\title{
Superposition Modulation Based Symmetric Relaying with Hybrid ARQ: Analysis and Optimization
}

\author{
Chaitanya Tumula V. K. and Erik G. Larsson
}

\section{Linköping University Pre Print}

N.B.: When citing this work, cite the original article.

C2011 IEEE. Personal use of this material is permitted. However, permission to reprint/republish this material for advertising or promotional purposes or for creating new collective works for resale or redistribution to servers or lists, or to reuse any copyrighted component of this work in other works must be obtained from the IEEE.

Chaitanya Tumula V. K. and Erik G. Larsson, Superposition Modulation Based Symmetric Relaying with Hybrid ARQ: Analysis and Optimization, 2011, accepted IEEE Transactions on Vehicular Technology. http://dx.doi.org/10.1109/TVT.2011.2162971

Preprint available at: Linköping University Electronic Press http://urn.kb.se/resolve?urn=urn:nbn:se:liu:diva-68288 


\title{
Superposition Modulation Based Symmetric Relaying with Hybrid ARQ: Analysis and Optimization
}

\author{
Tumula V. K. Chaitanya and Erik G. Larsson
}

\begin{abstract}
We present a retransmission scheme based on superposition modulation for the symmetric relaying scenario when the number of retransmissions for a data packet is limited. We consider both diversity combining based as well as code combining based retransmission schemes. Under the assumption that the receiver implements a mechanism that can use all accumulated received mutual information when decoding the message, we derive the packet error probability (PEP) expressions for the proposed retransmission scheme for the case when only one retransmission is allowed. Based on the PEP expressions derived, we provide a closed-form solution for the optimal superposition ratio (the fraction of power used for the relaying operation). Simulation results show that the proposed retransmission scheme offers significant gains compared to a retransmission scheme based on classical decode-and-forward (DF) relaying.
\end{abstract}

Index Terms-Cooperative communications, hybrid ARQ, superposition modulation, outage probability.

\section{INTRODUCTION}

Cooperative wireless systems [1], [2] have attracted much research interest recently due to their ability to provide transmit diversity through virtual antenna arrays. In a cooperative wireless system, users share their resources to forward each others' data to the destination. Several protocols have been developed in the literature for the operations at a relay. These are mainly classified into amplify-and-forward (AF) and decode-and-forward (DF) schemes [3]. For the symmetric relaying scenario (two users and a common destination, see Fig. 1), a bandwidth efficient transmission scheme based on DF relaying has been proposed in [4], which uses superposition modulation for transmitting the users data. This method of user cooperation has shown to be more bandwidth efficient than classical DF relaying with the same receiver complexity. This idea has been extended to superposition in the code domain in [5] and resulted in further improvements in performance, however this method requires carefully chosen codes and more complex iterative receivers. A performance analysis of iterative decoding for superposition modulation

Copyright (c) 2011 IEEE. Personal use of this material is permitted. However, permission to use this material for any other purposes must be obtained from the IEEE by sending a request to pubs-permissions@ieee.org.

T. V. K. Chaitanya and E. G. Larsson are with Linköping University, Dept. of Electrical Engineering (ISY), Division of Communication Systems, SE-581 83 Linköping, Sweden. (e-mail: \{tvk, erik.larsson $\} @$ isy.liu.se).

This work was supported in part by the Swedish Foundation for Strategic Research (SSF) and ELLIIT. E. G. Larsson is a Royal Swedish Academy of Sciences (KVA) Research Fellow supported by a grant from the Knut and Alice Wallenberg Foundation.

Parts of the material in this paper were presented at the IEEE VTC 2010 conference [22] based cooperative transmission scheme was presented in [6]. For an $N$-user cooperative multiple access system, cooperation using superposition modulation has been shown to achieve the optimal diversity-multiplexing gain [7]. In [8], the authors extended the idea to the case of incremental relaying, when feedback is available from the destination.

Fading in wireless channels results in the loss of data packets at the receiver. When a feedback channel from the receiver to the transmitter is available, hybrid automatic repeat request (H-ARQ) protocols are used in wireless networks to combat the effects of channel fading and thereby to provide reliable data transfer. There are different types of H-ARQ schemes proposed in literature, namely type I and type II HARQ schemes. More details about these schemes can be found in, for example, [11]. Many real-time wireless applications such as online gaming and video are delay sensitive and these applications have specific latency requirements [12]. In many current wireless standards like 3GPP - long term evolution (LTE), the number of retransmissions for a data packet is limited on the link layer. In this study, we assume that the maximum number of allowed retransmissions for a data packet is fixed to a given number, say $L$. That is, if the destination is not able to decode the data packet even after $L$ retransmissions, the data packet is dropped.

\section{A. Related Work and Contributions}

Conventional H-ARQ schemes can be easily extended to relaying systems, and several retransmission protocols exist in the literature for a single-relay network [13], [14] as well as for multi-relay networks [15], [16]. In these protocols, depending on the availability of feedback at the relay(s), either only the source or both the source and the relay(s) can manage a retransmission. An analytical framework for a cooperative DF relaying system with general hop-by-hop H-ARQ transmission was presented in [17], where the authors derived an upper bound on the block-error-rate assuming that the transmission from the source to the destination is hop-by-hop through the relay.

In this work, we discuss retransmission mechanisms for the symmetric relaying scenario. We propose a retransmission scheme based on the superposition modulation of [4] and compare its performance with that of a non-cooperative retransmission scheme and a retransmission scheme based on classical DF relaying [13]. For the proposed retransmission scheme, for simplicity, we limit ourselves to superposition in the modulation domain (signal space) as in [4]. However, 
we expect that the additional gains shown in [5] and [6] (with superposition in the code domain and with iterative decoding) over the original scheme in [4] (with superposition in the modulation domain) can also be achieved in case of retransmissions. We consider two cases in our study:

- Diversity Combining Case: The transmission from the relay and all the retransmissions carry the same information. At the destination, the receiver combines this information using maximum-ratio-combining (MRC) to decode the data packet.

- Code Combining Case: In this case, all the (re)transmissions carry new information. At the destination, the receiver does code combining to decode the data packet.

Under the assumption that the receiver implements a mechanism that can use all accumulated received mutual information when decoding the message, we derive analytical packet error probability expressions using outage probability analysis for $L=0$ and $L=1$. We obtain an exact closed-form expression for the diversity combining case and an approximate expression for the code combining case. For practical receivers, that cannot use all accumulated mutual information, these expressions represent lower bounds on the packet error probability.

In the initial work of [4], the superposition ratio (i.e., the amount of power allocated for the relaying operation) was optimized through simulations. An analysis and optimization of the superposition ratio for AF and DF based superposition modulation schemes has been presented in [9]. In the work of [9], under the assumption that each cooperating node have a priori knowledge of its partner's signal, the author used an equivalent multiple-access MIMO channel (with two transmitters) capacity region equations in the analysis to optimize the superposition ratio. The work in [10] focused on optimizing the superposition ratio with respect to maximizing the free distance of the resulting superimposed constellation. In this work, we optimize the superposition ratio by using the PEP expressions of both the diversity combining and the code combining methods for the following cases:

- The baseline relaying scheme using superposition modulation.

- The proposed retransmission scheme with $L=1$.

We also present results showing the optimal superposition ratio as a function of the spectral efficiency. Note that the techniques presented in this paper can also be used for analyzing nonrelaying scenarios such as, a H-ARQ transmission system in which an erroneous data packet is superimposed on a new data packet as in [20] or the multicast transmission setup in [21].

Finally we present simulation results comparing the performance of the proposed retransmission scheme with a retransmission scheme based on non-cooperative transmission and a retransmission scheme based on the DF relaying scheme of [13]. We also study the effect of varying the superposition ratio during the retransmissions.

This work is an extension of our conference paper [22], in which we discussed the retransmission scheme using diversity combining only. Herein, we also consider the code combining

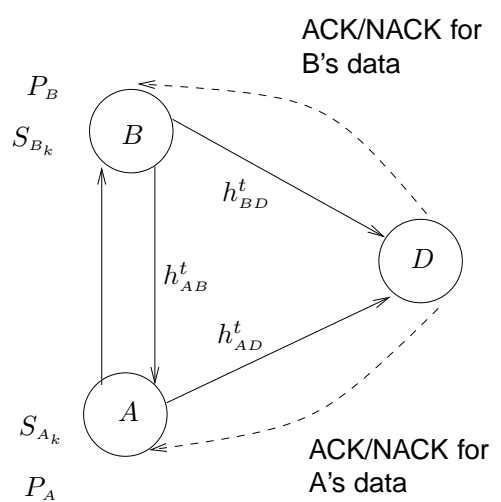

Figure 1. The symmetric relaying scenario of [4]

case as well as optimization of the superposition ratio. In this work, we also give the proofs for all derivations of the packet error probability expressions.

\section{B. Organization of the Paper}

In Section II, we discuss the three transmission methods considered in this paper. We present the new retransmission scheme based on superposition modulation for the symmetric relaying scenario in Section III. In Section IV, we derive packet error probability expressions for the proposed scheme. We discuss the optimization of the superposition ratio in Section V. Finally, we present simulation results in Section VI and conclusions in Section VII.

\section{SYSTEM MODEL}

In this section, we introduce the system model along with the three transmission methods considered in this paper. We are interested in the symmetric relaying scenario as shown in Fig. 1, in which two nodes A and B have data to send to a common destination $\mathrm{D}$. The motivation behind the symmetric scenario is that it does not require any additional relay(s) to help the users in transmitting their data. Users cooperate among themselves to realize the cooperative diversity. This scenario can be seen as an example of two users transmitting to a common base station in the uplink. Note that "symmetric" only refers to the topology of the network and does not mean that the A-B, A-D or B-D channel gains or their statistics have to be the same. We consider only time-division half-duplex systems with nodes transmitting over orthogonal channels (different time slots). We assume that the duration of time slots is the same for both users. Let $S_{n_{k}}$ denote the $k$ th data packet of node $n, k \in\{1,2, \ldots\}$ and $n \in\{A, B\}$. Each data packet consists of a set of modulation symbols drawn from a fixed constellation $\mathcal{S}$. We assume that each transmission consumes $T$ channel uses and that we transmit one modulation symbol per channel use. We also assume that we are interested in decoding node A's data at the destination (node B's data are treated in an analogous manner). Without loss of generality, for simplicity, we assume that the modulation symbols have unit energy. We consider a block fading channel in which the channel gains are constant during one packet transmission and change independently between 


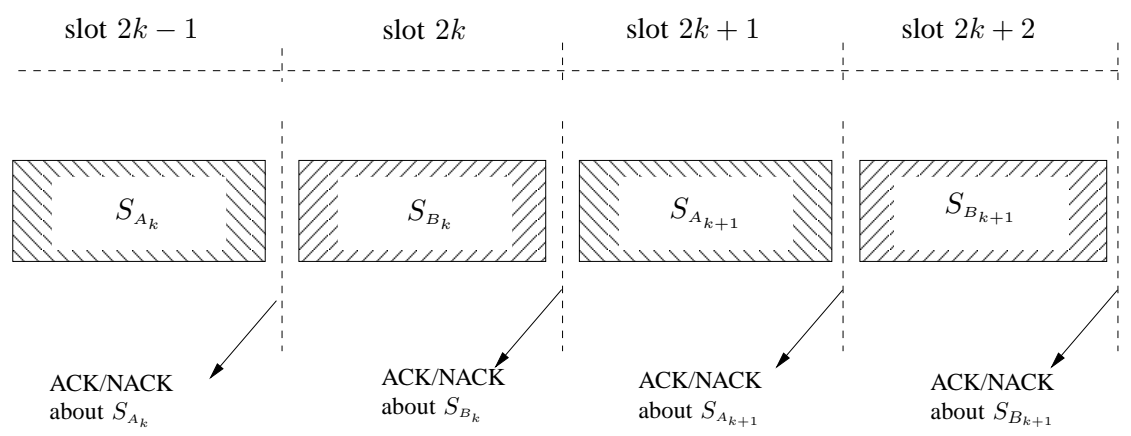

(a) Non-cooperative transmission.

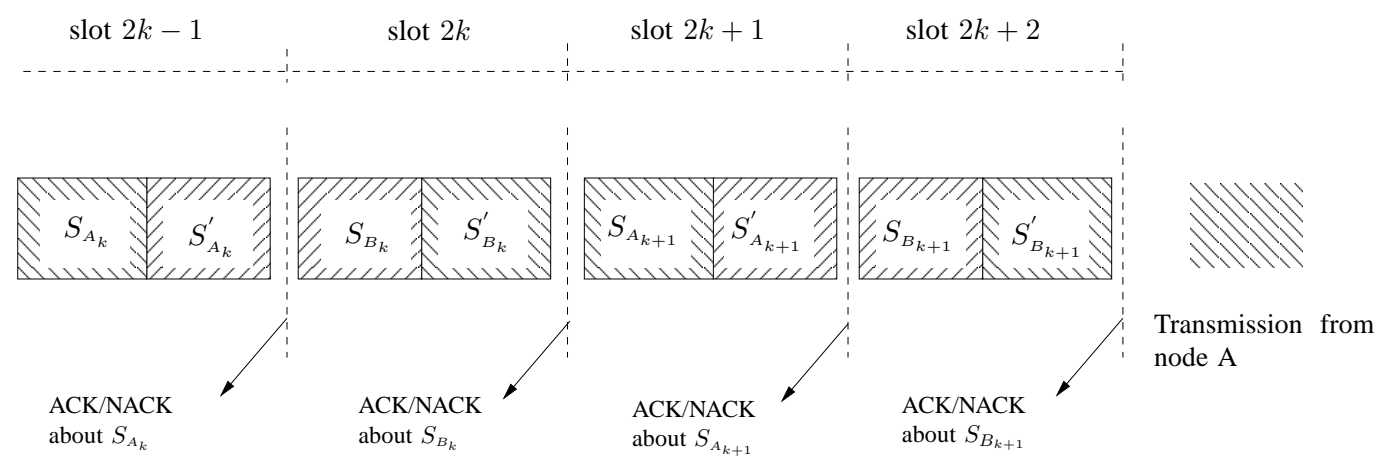

(b) Conventional DF relaying.

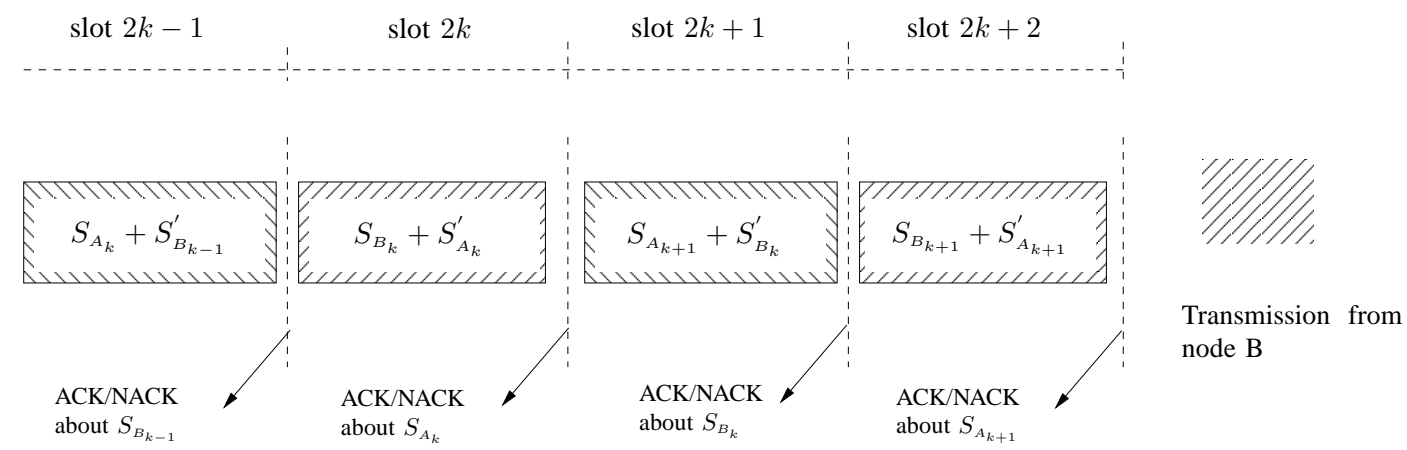

(c) Relaying with superposition modulation.

Figure 2. The three transmission methods considered for the symmetric relaying scenario [4].

the (re)transmissions. Let $h_{i j}^{l}$ denote the channel gain from $i \rightarrow j, i \in\{A, B\}$ and $j \in\{B, D\}$ during the $l$ th transmission of a data packet. We assume that these channel gains are independent and Rayleigh fading, with perfect channel state information (CSI) available at the corresponding receivers. We denote the average squared channel gains by $\lambda_{i j} \triangleq \mathbb{E}\left|h_{i j}^{l}\right|^{2}$, $\forall l$. We also assume that nodes A and B can transmit with average powers $P_{A}$ and $P_{B}$ respectively. Now we describe the transmission schemes considered in this paper, illustrated in Fig. 2.

\section{A. Non-cooperative Transmission}

With non-cooperative transmission as shown in Fig. 2(a), in time slot $2 k-1$, node A transmits its packet $S_{A_{k}}$ and in time slot $2 k$ node B transmits its data packet $S_{B_{k}}$. Since the nodes do not cooperate, this scheme cannot achieve diversity.

\section{B. Classical DF Relay Transmission}

In conventional DF relaying [1] as shown in Fig. 2(b), during the first half of time slot $2 k-1$, node A transmits its data packet $S_{A_{k}}$ and at the same time node B tries to decode $S_{A_{k}}$. If node B succeeds, it relays $S_{A_{k}}^{\prime}$ (potentially re-encoded using a different channel code) during the remaining half of 


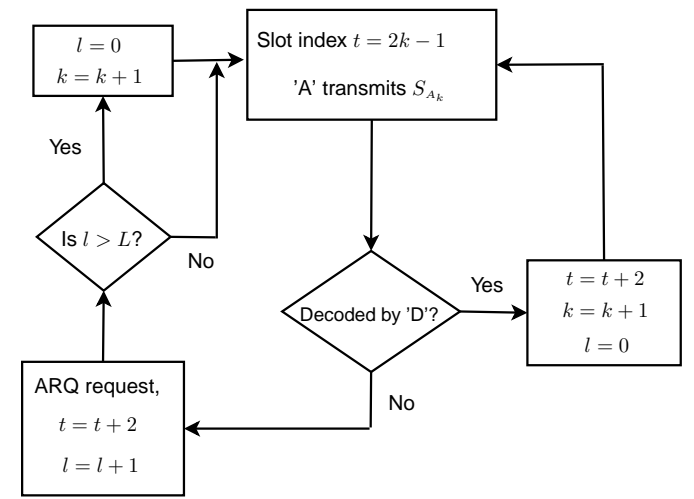

(a) Retransmission scheme for a non-cooperative system.

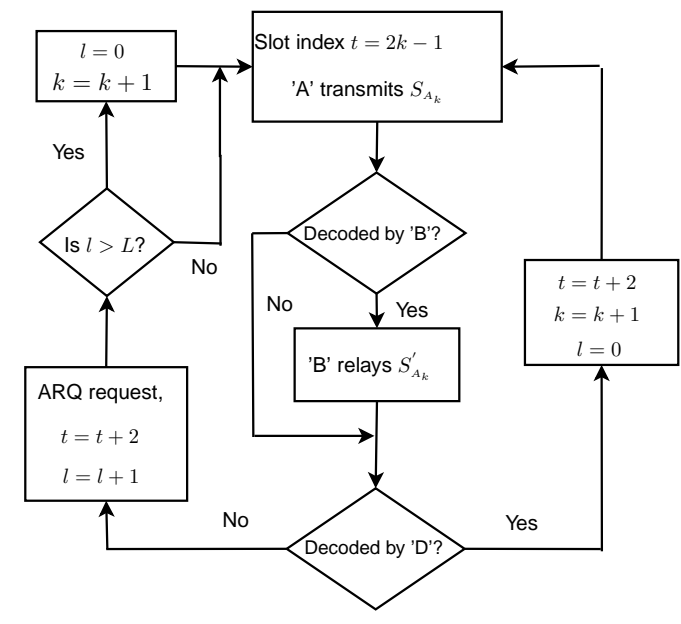

(b) Retransmission scheme based on DF relaying.

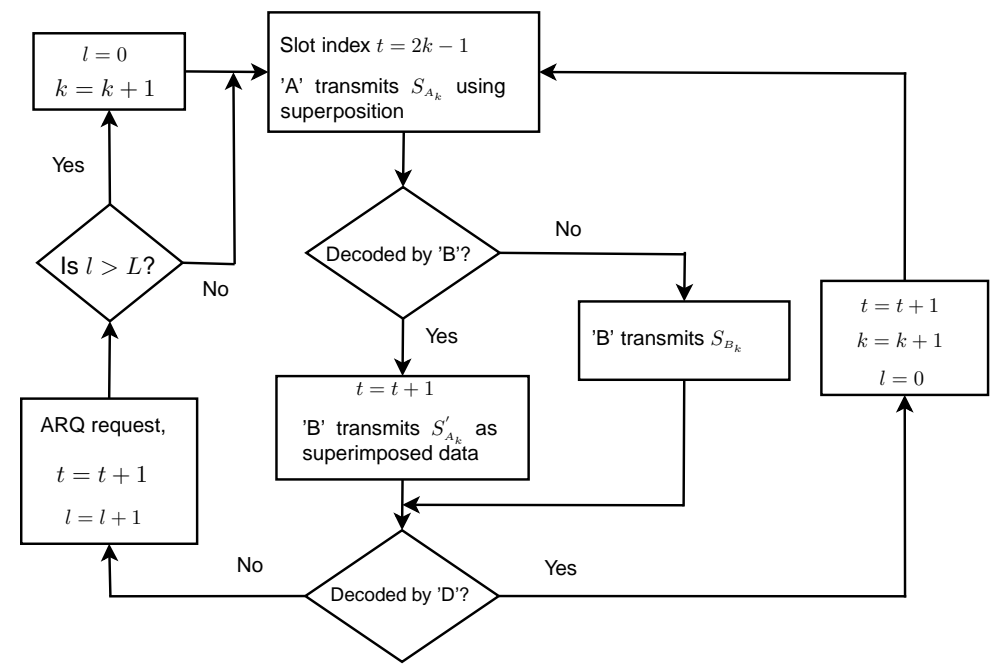

(c) Proposed retransmission scheme using superposition modulation based relaying.

Figure 3. Flowcharts for the retransmission schemes considered in this paper.

the time slot. During time slot $2 k$, the procedure is repeated for the packet $S_{B_{k}}$. When the inter-user channel is strong, each data packet is transmitted from both the users and hence this scheme achieves a diversity order of 2 . A precise analysis of DF relaying is given in [23].

\section{Superposition Modulated Cooperative Transmission}

In the superposition modulation based cooperation of [4], along with its own data, each node forwards the data overheard from other node in the previous time slot using superposition modulation. The amount of superposition is varied by the superposition ratio $\gamma$, which is the fraction of the total transmit power allocated for the partner's data. As shown in Fig. 2(c), during time slot $2 k-1$, node A transmits its own packet $S_{A_{k}}$ superimposed with node B's packet $S_{B_{k-1}}^{\prime}$ which it has received during the previous time slot. The received signals at nodes $\mathrm{D}$ and $\mathrm{B}$ during time slot $2 k-1$ can be written as:

$$
\begin{aligned}
& y_{D_{2 k-1}}=\sqrt{P_{A}} h_{A D}^{2 k-1}\left(\sqrt{1-\gamma} S_{A_{k}}+\sqrt{\gamma} S_{B_{k-1}^{\prime}}^{\prime}\right)+w_{D_{2 k-1}} \\
& y_{B_{2 k-1}}=\sqrt{P_{A}} h_{A B}^{2 k-1}\left(\sqrt{1-\gamma} S_{A_{k}}+\sqrt{\gamma} S_{B_{k-1}}^{\prime}\right)+w_{B_{2 k-1}}
\end{aligned}
$$

where $w_{D_{2 k-1}}$ and $w_{B_{2 k-1}}$ denote the additive noise at nodes $\mathrm{D}$ and B during time slot $2 k-1$. Node B decodes $S_{A_{k}}$ using a maximum a posteriori (MAP) detector as described in [4]. Assuming successful decoding of $S_{A_{k}}$, node B re-encodes it to obtain the packet $S_{A_{k}}^{\prime}$ and transmits it using superposition modulation along with its own data packet $S_{B_{k}}$ in time slot $2 k$. The received signals at nodes $\mathrm{D}$ and A during time slot $2 k$ can be written as:

$$
\begin{aligned}
& y_{D_{2 k}}=\sqrt{P_{B}} h_{B D}^{2 k}\left(\sqrt{1-\gamma} S_{B_{k}}+\sqrt{\gamma} S_{A_{k}}^{\prime}\right)+w_{D_{2 k}} \\
& y_{A_{2 k}}=\sqrt{P_{B}} h_{A B}^{2 k}\left(\sqrt{1-\gamma} S_{B_{k}}+\sqrt{\gamma} S_{A_{k}}^{\prime}\right)+w_{A_{2 k}}
\end{aligned}
$$

where $w_{D_{2 k}}, w_{A_{2 k}}$ denote the additive noise at nodes $\mathrm{D}$ and $\mathrm{A}$ during time slot $2 k$. The destination node recovers $S_{A_{k}}$ using a MAP detector by operating on the information received during the two successive slots $2 k-1$ and $2 k$ [4]. Without loss of generality, we assume that the noise samples per channel use at the nodes $\mathrm{A}, \mathrm{B}$ and $\mathrm{D}$ are i.i.d. with distribution $\mathcal{C N}(0,1)$. 


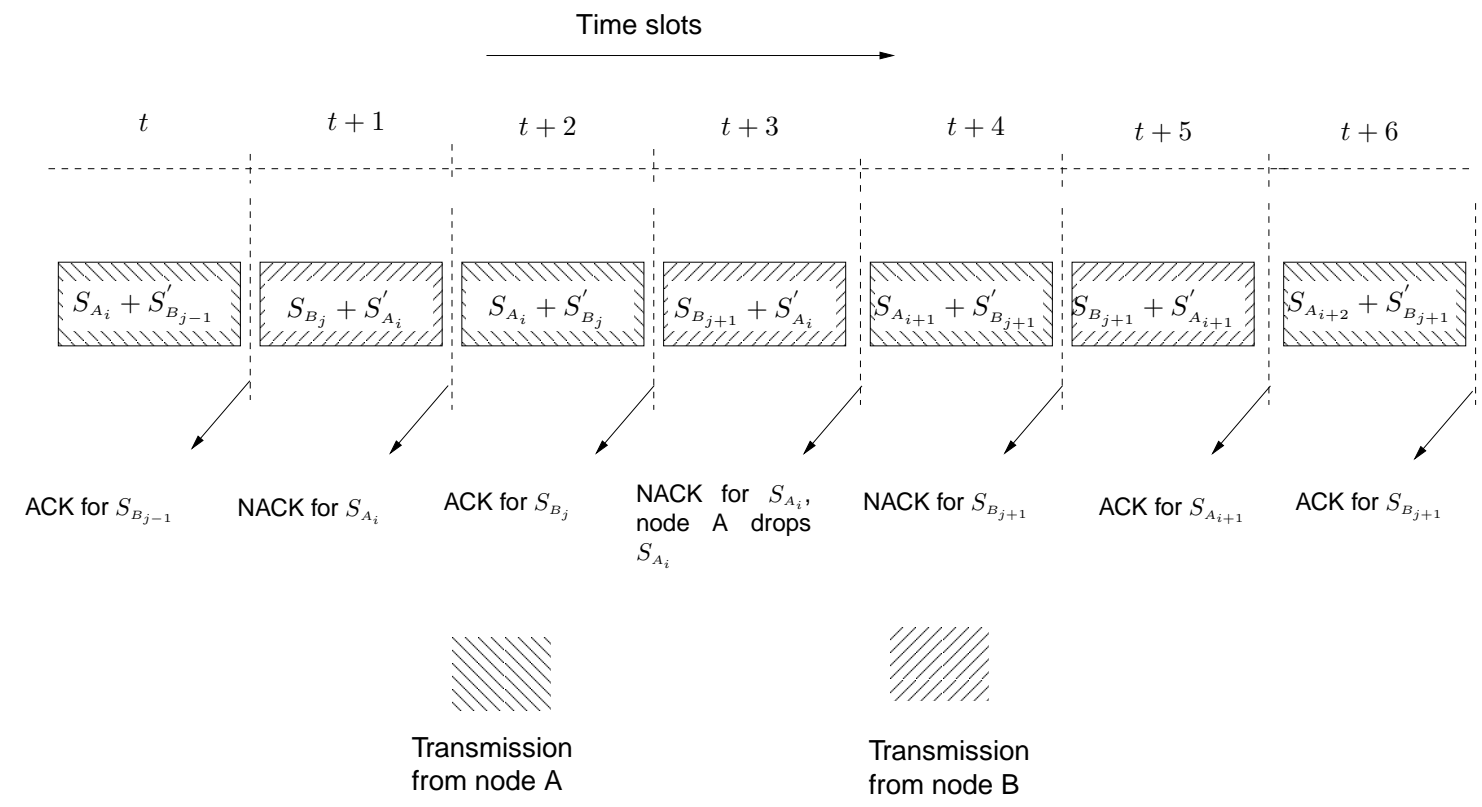

Figure 4. An example scenario of the proposed retransmission scheme based on superposition modulation based relaying with $L=1$.

\section{RETRANSMISSION SCHEMES FOR THE SYMMETRIC RELAYING SCENARIO}

In this section, we present our new retransmission scheme based on superposition modulation for the symmetric relaying scenario. Before that, we briefly describe a retransmission scheme for the non-cooperative transmission as well as a retransmission scheme based on DF relaying. We chose these base retransmission schemes from [13] and adapted them to the symmetric relaying scenario. We consider type-II H-ARQ schemes with both diversity combining and code combining. In case of the H-ARQ scheme based on diversity combining (i.e., $S_{A_{k}}^{\prime}=S_{A_{k}}$ and the same $S_{A_{k}}$ is retransmitted if the original transmission is in error), the receiver performs MRC to decode the data packet. For the code combining case, we assume that the duration of the time slots for retransmissions is the same as that of the first transmission. We also assume that the ACK/NACK signals sent by D are received both by node A and node $\mathrm{B}$ and that the feedback channel is instantaneous and error-free. The source node (A in the present case) maintains a retransmission index counter $l$ and increments it for each new retransmission. If $l$ becomes greater than $L$, it drops the current packet and transmits the next one.

\section{A. Retransmission Scheme Based on Non-cooperative Trans- mission}

In a conventional non-cooperative retransmission protocol, node A retransmits the erroneously received packet in its allocated slots (odd-numbered time slots in Fig. 2) until it receives an ACK signal or it reaches the maximum number of retransmissions limit. Note that node B does not contribute to the retransmission mechanism here. A flowchart for the noncooperative retransmission scheme is shown in Fig. 3(a).

\section{B. Retransmission Scheme Based on DF Relaying}

Fig. 3(b) shows a flowchart of the retransmission scheme based on DF relaying that we consider. After getting the
NACK signal from node D for its packet $S_{A_{k}}$ transmitted during time slot $2 k-1$, node $\mathrm{A}$ retransmits it during the first half of the odd-numbered time slots and node B also relays $S_{A_{k}}^{\prime}$ during the other half of the odd-numbered time slots. In this retransmission protocol both node A and node B retransmit the data packet of node A which was in error.

\section{Proposed Retransmission Scheme for the Symmetric Relay- ing Scenario}

Fig. 3(c) summarizes the proposed retransmission scheme using superposition modulation based relaying. If the packet $S_{A_{k}}$ is in error, node A will retransmit the same packet during the odd-numbered time slots and superimpose node B's data that it received during the previous slot. During the evennumbered time slots, node B will transmit its own packet (a new packet or a retransmitted packet) as well as node A's packet $S_{A_{k}}^{\prime}$ using superposition modulation. This procedure is continued until $S_{A_{k}}$ is successfully decoded by node D or $l$ reaches its upper limit $L$.

An example scenario of the proposed retransmission scheme with only one allowed retransmission is shown in Fig. 4. As shown in figure, when node A receives a NACK signal for the packet $S_{A_{i}}$ at the end of time slot $t+1$, during time slot $t+2$, node A retransmits $S_{A_{i}}$ along with node B's packet $S_{B_{j}}^{\prime}$ using superposition modulation. During time slot $t+3$, node $\mathrm{B}$ also relays $S_{A_{i}}^{\prime}$ using superposition modulation. The destination tries to decode $S_{A_{i}}$ using the information it received during time slots $t$ to $t+3$. If it has not succeeded in decoding, it again sends a NACK signal for $S_{A_{i}}$ as shown in the figure. After receiving the NACK signal again, node A drops the packet $S_{A_{i}}$ and transmits $S_{A_{i+1}}$ in time slot $t+4$. In general the packet indices of the users need not be synchronized during the superposition operation.

The proposed retransmission scheme with superposition modulation based relaying has the same complexity as that of the retransmission scheme with DF relaying. In both retransmission schemes, decoding operations need to be performed at 
the cooperating node as well as at the destination node. Even though joint detection is performed at the destination node for the proposed method, the complexity of soft demodulation is the same as that of the DF based scheme. This is due to the fact that the target spectral efficiency (number of bits per channel use) of the DF based scheme is twice that of the proposed method.

If one can design codes similar to the ones in [5] for the scenarios involving retransmissions considered herein, then superposition in the code domain could be used instead of superposition in the modulation domain. The complexity at the receiver for the code-domain superposition scheme would depend on the specific type of channel codes used. We therefore have chosen not to include quantitative complexity comparisons between the proposed retransmission scheme with modulation domain superposition and code domain superposition.

In the retransmission schemes based on DF relaying and superposition modulation which use node B for relaying, we assume that the source node sends a "new packet" flag bit with each data packet to enable node B to distinguish between a new packet and a retransmitted packet. Based on this information, node $\mathrm{B}$ will try to decode the retransmitted packet from node A only if it was not successfully decoded during the previous (re)transmissions.

\section{PACKet ERror Probability Analysis}

In this section, we present analytical expressions for the outage probability of the proposed retransmission scheme based on superposition modulation. In superposition modulation based relaying, the destination receives information about a user's data from both the nodes in two successive time slots. We assume that the receiver can use all data received in the past and that it has a mechanism to accumulate all the received mutual information about a data packet for performing the decoding operation. The iterative detection and decoding with interference cancellation approach of [6] is one way of getting close to this in practice. Under this assumption, the result is that the SNR at node D for node A's data during two successive slots is $P_{A}^{\prime}\left|h_{A D}\right|^{2}$ and $P_{B}^{\prime}\left|h_{B D}\right|^{2}$, where $P_{A}^{\prime} \triangleq P_{A}(1-\gamma)$ and $P_{B}^{\prime} \triangleq P_{B} \gamma$. Note that for practical systems that cannot make use of all mutual information accumulated by the receiver, the packet error probability expressions that we derive in what follows represent lower bounds.

A similar interference-free separation of users data assumption was also made in [10]. The paper [10] assumed that perfect Costa precoding (also known as dirty paper coding, DPC) [18] could be performed at the cooperating nodes. However this assumption appears to be invalid, since one cannot use the assumption of perfect DPC for the symmetric relaying scenario with superposition modulation and achieve the SNRs $P_{A}^{\prime}\left|h_{A D}\right|^{2}$ and $P_{B}^{\prime}\left|h_{B D}\right|^{2}$ in two successive time slots. The reason is that DPC is applicable only in scenarios where the destination is not interested in decoding the interference data, whereas in symmetric relaying using superposition modulation, the receiver is also interested in decoding interference (partners') data.
Under the assumptions stated above, we derive the packet error probability $\left(\mathrm{PEP}^{L}\right)$ expressions for the proposed scheme when $L=0$ (after the first transmission) and $L=1$ (allowing for one retransmission). For mathematical tractability, we assume that node A always uses superposition modulation during its transmission (that is, node B's data is always successfully decoded at node A). We also assume that the nodes use the same superposition ratio $(\gamma)$ value during the retransmissions.

We use outage events to characterize whether node D sends an NACK/ACK signal after a transmission. A transmission link with a received SNR of $\beta$ and a target spectral efficiency of $R$ bits per channel use (bpcu) is in outage if the instantaneous spectral efficiency ${ }^{1}$ given by $I \triangleq \log _{2}(1+\beta)$ is smaller than $R$. The probability of outage can be written as:

$$
\mathrm{P}_{\text {out }} \triangleq \operatorname{Pr}(I<R)
$$

Let $I_{x y}^{l}$ denote the average spectral efficiency along the path $x \rightarrow y$ during the $l$ th retransmission and let $I_{M R C}\left(x y^{l}, z y^{m}\right)$ denote the average spectral efficiency at node $y$ after the MRC as a function of $x y^{l}$ and $z y^{m}$, i.e., the information it received along the path $x \rightarrow y$ in the $l$ th transmission and along the path $z \rightarrow y$ in the $m$ th transmission respectively. Note that $l=0$ corresponds to the first transmission. Under the assumption described earlier in this section, as an example, we write $I_{A D}^{l}=\log _{2}\left(1+P_{A}^{\prime} \alpha_{A D}^{l}\right)$, where $\alpha_{A D}^{l}=\left|h_{A D}^{l}\right|^{2}, l=$ $0,1, \ldots, L$, with

$$
f_{\alpha_{i j}^{l}}(t)=\frac{1}{\lambda_{i j}} \exp \left(-\frac{t}{\lambda_{i j}}\right), t \geq 0,
$$

with $i \in\{A, B\}$ and $j \in\{B, D\}$.

\section{A. Analysis for the Diversity Combining Case}

1) Derivation of $\mathrm{PEP}_{\mathrm{div}}^{0}$ : When there are no retransmissions allowed $(L=0)$, the packet error probability for the diversity combining case can be written in terms of the probability of two disjoint events. First event being that both the links $A \rightarrow B$ and $A \rightarrow D$ are in outage simultaneously and the second event being that even though $A \rightarrow B$ is not in outage, the destination is still in outage even after receiving data along $A \rightarrow D$ and $B \rightarrow D$. By using the fact that $\alpha_{A D}$, $\alpha_{A B}$ and $\alpha_{B D}$ are independent, we can write:

$$
\begin{aligned}
\mathrm{PEP}_{\text {div }}^{0}= & \operatorname{Pr}\left(I_{A B}^{0}<R\right) \operatorname{Pr}\left(I_{A D}^{0}<R\right)+ \\
& \operatorname{Pr}\left(I_{A B}^{0}>R\right) \operatorname{Pr}\left(I_{M R C}\left(A D^{0}, B D^{0}\right)<R\right)
\end{aligned}
$$

where $R$ is the target spectral efficiency in bpcu and

$$
I_{M R C}\left(A D^{0}, B D^{0}\right)=\log _{2}\left(1+P_{A}^{\prime} \alpha_{A D}^{0}+P_{B}^{\prime} \alpha_{B D}^{0}\right)
$$

If $N_{I}$ is the number of information bits, then $R=\frac{N_{I}}{T}$. Using the result in Appendix A, (4) can be simplified to arrive at (5) shown on top of the next page, in which $X_{A B} \triangleq \frac{1-2^{R}}{P_{A}^{\prime} \lambda_{A B}}$,

\footnotetext{
${ }^{1}$ This assumes an infinite block length and a Gaussian code book. However for many practical schemes with adaptive modulation and coding, the instantaneous spectral efficiency can be expressed as $I \approx \log _{2}(1+\zeta \beta)$, where $\zeta$ is a penalty factor (distance from the Shannon capacity) [19].
} 


$$
\mathrm{PEP}_{\text {div }}^{0}= \begin{cases}{\left[1-\exp \left(X_{A D}\right)\right]\left[1-\exp \left(X_{A B}\right)\right]+\exp \left(X_{A B}\right) \times} & \\ {\left[1-\exp \left(X_{B D}\right)-\frac{\exp \left(X_{A D}\right)}{\delta}\left(1-\exp \left(X_{B D} \delta\right)\right)\right],} & \text { if } \delta \neq 0 \\ {\left[1-\exp \left(X_{A D}\right)\right]\left[1-\exp \left(X_{A B}\right)\right]+\exp \left(X_{A B}\right) \times} & \\ {\left[1-\exp \left(X_{B D}\right)+X_{B D} \exp \left(X_{A D}\right)\right],} & \text { if } \delta=0\end{cases}
$$

$$
\begin{aligned}
\operatorname{PEP}_{\text {div }}^{1}= & \operatorname{Pr}\left(I_{M R C}\left(A B^{0}, A B^{1}\right)<R\right) \operatorname{Pr}\left(I_{M R C}\left(A D^{0}, A D^{1}\right)<R\right)+\operatorname{Pr}\left(I_{A B}^{0}<R, I_{M R C}\left(A B^{0}, A B^{1}\right)>R\right) \times \\
& \operatorname{Pr}\left(I_{M R C}\left(A D^{0}, A D^{1}, B D^{1}\right)<R\right)+\operatorname{Pr}\left(I_{A B}^{0}>R\right) \operatorname{Pr}\left(I_{M R C}\left(A D^{0}, A D^{1}, B D^{0}, B D^{1}\right)<R\right)
\end{aligned}
$$

$$
\operatorname{PEP}_{\text {div }}^{1}= \begin{cases}{\left[1-\exp \left(X_{A D}\right)\left(1-X_{A D}\right)\right]\left[1-\exp \left(X_{A B}\right)\left(1-X_{A B}\right)\right]-} \\ X_{A B} \exp \left(X_{A B}\right)\left[1-\exp \left(X_{B D}\right)+\frac{\exp \left(X_{A D}\right)\left(X_{A D}-1\right)}{\delta}\left(1-\exp \left(X_{B D} \delta\right)\right)+\right. \\ \left.(1-\delta) \exp \left(X_{A D}\right)\left(\exp \left(X_{B D} \delta\right)\left(\frac{X_{B D}}{\delta}-\frac{1}{\delta^{2}}\right)+\frac{1}{\delta^{2}}\right)\right]+ \\ \exp \left(X_{A B}\right)\left[1-\exp \left(X_{B D}\right)\left(1-X_{B D}\right)+\exp \left(X_{A D}\right)\left(X_{A D}-1\right) \times\right. \\ \left(\exp \left(X_{B D} \delta\right)\left(\frac{X_{B D}}{\delta}-\frac{1}{\delta^{2}}\right)+\frac{1}{\delta^{2}}\right)+ \\ \left.(1-\delta) \exp \left(X_{A D}\right)\left(\exp \left(X_{B D} \delta\right)\left(-\frac{X_{B D}^{2}}{\delta}+\frac{2 X_{B D}}{\delta^{2}}-\frac{2}{\delta^{3}}\right)+\frac{2}{\delta^{3}}\right)\right], & \text { if } \delta \neq 0 \\ {\left[1-\exp \left(X_{A D}\right)\left(1-X_{A D}\right)\right]\left[1-\exp \left(X_{A B}\right)\left(1-X_{A B}\right)\right]-} & \text { if } \delta=0 \\ X_{A B} \exp \left(X_{A B}\right)\left[1-\exp \left(X_{B D}\right)-\exp \left(X_{A D}\right)\left(X_{A D}-1\right) X_{B D}+\frac{\exp \left(X_{A D}\right) X_{A D} X_{B D}}{2}\right]+ & \\ \exp \left(X_{A B}\right)\left[1-\exp \left(X_{B D}\right)\left(1-X_{B D}\right)+\right. & \\ \left.\exp \left(X_{A D}\right)\left(X_{A D}-1\right) \frac{X_{B D}^{2}}{2}-\frac{\left.\exp \left(X_{A D}\right) X_{A D} X_{B D}^{2}\right],}{3}\right] & \end{cases}
$$

$$
\begin{aligned}
\operatorname{PEP}_{\mathrm{cc}}^{1}= & \operatorname{Pr}\left(I_{A B}^{0}+I_{A B}^{1}<R\right) \operatorname{Pr}\left(I_{A D}^{0}+I_{A D}^{1}<R\right)+\operatorname{Pr}\left(I_{A B}^{0}<R, I_{A B}^{0}+I_{A B}^{1}>R\right) \times \\
& \operatorname{Pr}\left(I_{A D}^{0}+I_{A D}^{1}+I_{B D}^{1}<R\right)+\operatorname{Pr}\left(I_{A B}^{0}>R\right) \operatorname{Pr}\left(I_{A D}^{0}+I_{A D}^{1}+I_{B D}^{0}+I_{B D}^{1}<R\right)
\end{aligned}
$$

$X_{B D} \triangleq \frac{1-2^{R}}{P_{B}^{\prime} \lambda_{B D}}, X_{A D} \triangleq \frac{1-2^{R}}{P_{A}^{\prime} \lambda_{A D}}$ and $\delta \triangleq 1-\frac{P_{B}^{\prime} \lambda_{B D}}{P_{A}^{\prime} \lambda_{A D}}$. By using a series expansion, it can be shown that

$$
\mathrm{PEP}_{\mathrm{div}}^{0}=X_{A D} X_{A B}+\frac{1}{2} X_{A D} X_{B D}+O\left(\frac{1}{P^{3}}\right)
$$

From (6), we see that the superposition modulation based relaying scheme has a diversity order of 2 .

2) Derivation of $\mathrm{PEP}_{\text {div }}^{1}$ : When only one retransmission is allowed $(L=1)$, the packet error probability for the diversity combining case can be expressed in terms three disjoint events that the destination is still in outage depending on whether the link $A \rightarrow B$ is i) in outage after one retransmission or ii) in outage after first transmission but not in outage after one retransmission or iii) not in outage after first transmission. Since $\alpha_{A D}, \alpha_{A B}$ and $\alpha_{B D}$ are independent, we can write this outage probability as shown in (7) on top of this page. By simplifying (7) as in Appendix B, we arrive at the expression in (8) shown on top of this page. We can show that using a series expansion, $\mathrm{PEP}_{\mathrm{div}}^{1}$ can be written as

$\mathrm{PEP}_{\mathrm{div}}^{1}=\frac{1}{4} X_{A D}^{2} X_{A B}^{2}+\frac{1}{6} X_{A B} X_{A D}^{2} X_{B D}+\frac{1}{24} X_{B D}^{2} X_{A D}^{2}+O\left(\frac{1}{P^{5}}\right)$

From (9), we see that with one additional retransmission, we can achieve a diversity order of 4 with the proposed superposition modulation based retransmission scheme.

\section{B. Analysis for the Code Combining Case}

1) Derivation of $\mathrm{PEP}_{\mathrm{cc}}^{0}$ : In case of code combining at the destination, when there are no retransmissions allowed, the packet error probability can be written similarly based on the disjoint events described in section IV-A1 as:

$$
\begin{aligned}
\operatorname{PEP}_{\mathrm{cc}}^{0}= & \operatorname{Pr}\left(I_{A B}^{0}<R\right) \operatorname{Pr}\left(I_{A D}^{0}<R\right)+ \\
& \operatorname{Pr}\left(I_{A B}^{0}>R\right) \operatorname{Pr}\left(I_{A D}^{0}+I_{B D}^{0}<R\right)
\end{aligned}
$$

Simplifying $\operatorname{Pr}\left(I_{A D}^{0}+I_{B D}^{0}<R\right)$ as in Appendix C, $\mathrm{PEP}_{\mathrm{cc}}^{0}$ can be written as:

$$
\mathrm{PEP}_{\mathrm{cc}}^{0}=X_{A D} X_{A B}+\frac{2^{R} R \ln 2-2^{R}+1}{P_{A}^{\prime} P_{B}^{\prime} \lambda_{A D} \lambda_{B D}}+O\left(\frac{1}{P^{3}}\right)
$$

This scheme also achieves a diversity order of 2 .

2) Derivation for $\mathrm{PEP}_{\mathrm{cc}}^{1}$ : When only one retransmission is allowed, the packet error probability for the code combining case can be written similarly based on the disjoint events described in section IV-A2 as in (12) shown at the top of this page. Simplifying (12) as in Appendix $\mathrm{D}, \mathrm{PEP}_{\mathrm{cc}}^{1}$ can be written as in (13) shown on top of the next page. The expression in (13) shows that the proposed retransmission scheme achieves a diversity order of 4 for the code combining case. 


$$
\begin{aligned}
\mathrm{PEP}_{\mathrm{cc}}^{1}= & \frac{\left(2^{R} R \ln 2-2^{R}+1\right)^{2}}{\left(P_{A}^{\prime}\right)^{4} \lambda_{A D}^{2} \lambda_{A B}^{2}}+\frac{\left(2^{R}-1\right)\left(2^{R}(R \ln 2)^{2}-2\left(2^{R} R \ln 2-2^{R}+1\right)\right)}{2\left(P_{A}^{\prime}\right)^{3} P_{B}^{\prime} \lambda_{A D}^{2} \lambda_{B D} \lambda_{A B}}+ \\
& \frac{2^{R}(R \ln 2)^{3}-3\left(2^{R}(R \ln 2)^{2}\right)+6\left(2^{R} R \ln 2-2^{R}+1\right)}{6\left(P_{A}^{\prime} P_{B}^{\prime} \lambda_{A D} \lambda_{B D}\right)^{2}}+O\left(\frac{1}{P^{5}}\right)
\end{aligned}
$$

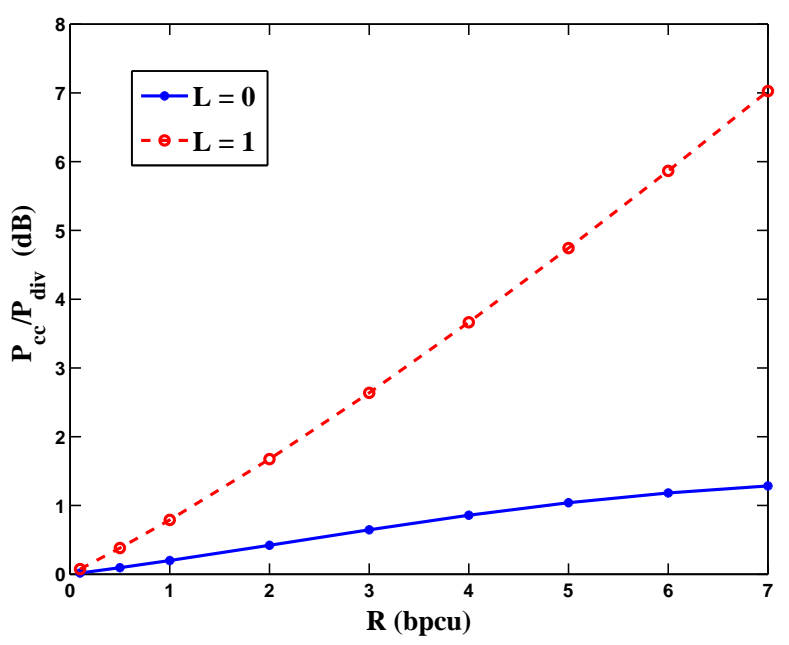

Figure 5. Power gain in $\mathrm{dB}$ for the code combining case over the diversity combining case as a function of $R$. We have used $\lambda_{A D}=\lambda_{A D}=\lambda_{B D}$.

\section{Maximal Ratio Combining vs Code Combining}

In this subsection, we compare the analytical packet error probability expressions derived in Sections IV-A and IV-B. By assuming that $P_{A}=P_{B}=P_{\text {div }}$ for the the diversity combining case and $P_{A}=P_{B}=P_{\mathrm{cc}}$ for the code combining case, we are interested in computing the power gain for the code combining case over the diversity combining case. For $L=0$, by neglecting $O\left(\frac{1}{P^{3}}\right)$ terms, we compute $\frac{P_{\mathrm{cc}}}{P_{\mathrm{div}}}$ by equating (6) and (11). Similarly for $L=1$, the power ratio is computed by neglecting $O\left(\frac{1}{P^{5}}\right)$ terms and equating (9) and (13). Fig. 5 shows the power gain for code combining scheme over the diversity combining scheme as a function of $R$. As we see from the figure, when there is one additional retransmission, the gain for code combining over diversity combining is significant.

\section{Optimization of The SUPERPosition Ratio}

In this section, we discuss the optimization of $\gamma$ based on the PEP expressions derived in Section IV.

\section{A. For $L=0$}

Based on the PEP expressions for the diversity combining and the code combining cases, one can numerically optimize the superposition ratio by considering minimization of $\mathrm{PEP}_{\text {div }}^{0}$ and $\mathrm{PEP}_{\mathrm{cc}}^{0}$ subject to $0 \leq \gamma \leq 0.5$. From the approximate PEP expressions in (6) and (11) and neglecting the $O\left(\frac{1}{P^{3}}\right)$ terms, we have the following optimization problem:

$$
\min _{0 \leq \gamma \leq 0.5} f_{1}(\gamma)=\frac{K_{1}}{(1-\gamma)^{2}}+\frac{K_{2}}{(1-\gamma) \gamma}
$$

where $K_{1} \triangleq \frac{\left(2^{R}-1\right)^{2}}{P_{A}^{2} \lambda_{A D} \lambda_{A B}}$ for both the diversity combining (D. C.) and the code combining (C. C.) methods and $K_{2}$ is given by:

$$
K_{2} \triangleq \begin{cases}\frac{\left(2^{R}-1\right)^{2}}{2 P_{A} P_{B} \lambda_{A D} \lambda_{B D}} & \text { for D. C. } \\ \frac{2^{R} R \ln 2-2^{R}+1}{P_{A} P_{B} \lambda_{A D} \lambda_{B D}} & \text { for C. C. }\end{cases}
$$

A closed form solution to this optimization problem can be obtained by taking the derivative of the objective function with respect to $\gamma$ and equating it to zero as follows:

$$
\begin{aligned}
\frac{\partial f_{1}(\gamma)}{\partial \gamma} & =\frac{2 K_{1}}{(1-\gamma)^{3}}+K_{2}\left[\frac{1}{\gamma(1-\gamma)^{2}}-\frac{1}{\gamma^{2}(1-\gamma)}\right]=0 \\
& \Longrightarrow \frac{2 K_{1}}{(1-\gamma)^{3}}+\frac{K_{2}(2 \gamma-1)}{\gamma^{2}(1-\gamma)^{2}}=0 \\
& \Longrightarrow 2 K_{1} \gamma^{2}+K_{2}(2 \gamma-1)(1-\gamma)=0 \\
& \Longrightarrow \gamma^{2}\left(2 K_{1}-2 K_{2}\right)+3 K_{2} \gamma-K_{2}=0
\end{aligned}
$$

The optimal superposition ratio for $L=0$ is given by:

$$
\gamma_{\mathrm{opt}}^{0}= \begin{cases}\frac{-3 K_{2}+\sqrt{8 K_{1} K_{2}+K_{2}^{2}}}{4\left(K_{1}-K_{2}\right)} & \text { if } K_{1} \neq K_{2} \\ \frac{1}{3} & \text { if } K_{1}=K_{2}\end{cases}
$$

For diversity combining, we see that the optimal superposition ratio for $K_{1} \neq K_{2}$ is independent of $R$ as both numerator and denominator values scale by the same factor in (15) for fixed values of average channel gains and the average power values. Note that this conclusion is not valid for the code combining case.

\section{B. For $L=1$}

Using the approximate PEP expressions from (9) and (13) and neglecting the $O\left(\frac{1}{P^{5}}\right)$ terms, we can formulate the optimization problem as ${ }^{2}$ :

$$
\min _{0 \leq \gamma \leq 0.5} f_{2}(\gamma)=\frac{C_{1}}{(1-\gamma)^{4}}+\frac{C_{2}}{(1-\gamma)^{3} \gamma}+\frac{C_{3}}{(1-\gamma)^{2} \gamma^{2}}
$$

where

$$
C_{1} \triangleq \begin{cases}\frac{\left(2^{R}-1\right)^{4}}{4 P_{A}^{4} \lambda_{A D}^{2} \lambda_{A B}^{2}} & \text { for D. C. } \\ \frac{\left(2^{R} R \ln 2-2^{R}+1\right)^{2}}{P_{A}^{4} \lambda_{A D}^{2} \lambda_{A B}^{2}} & \text { for C. C. }\end{cases}
$$

${ }^{2}$ In this work, we assumed that the same superposition ratio value $\gamma$ is used for all the transmissions. This assumption may be generalized. If $\gamma_{1}$ and $\gamma_{2}$ denote the superposition ratio values during the first transmission and the retransmission for the case of $L=1$, one can write the corresponding optimization problem as:

$$
\begin{aligned}
\min _{0 \leq \gamma_{1}, \gamma_{2} \leq 0.5} & \frac{C_{1}}{\left(1-\gamma_{1}\right)^{2}\left(1-\gamma_{2}\right)^{2}}+\frac{C_{2}}{\left(1-\gamma_{1}\right)^{2}\left(1-\gamma_{2}\right) \gamma_{2}}+ \\
& \frac{C_{3}}{\left(1-\gamma_{1}\right) \gamma_{1}\left(1-\gamma_{2}\right) \gamma_{2}}
\end{aligned}
$$




$$
C_{2} \triangleq \begin{cases}\frac{\left(2^{R}-1\right)^{4}}{6 P_{A}^{3} P_{B} \lambda_{A D}^{2} \lambda_{B D} \lambda_{A B}} & \text { for D. C. } \\ \frac{\left(2^{R}-1\right)\left(2^{R}(R \ln 2)^{2}-2\left(2^{R} R \ln 2-2^{R}+1\right)\right)}{2 P_{A}^{3} P_{B} \lambda_{A D}^{2} \lambda_{B D} \lambda_{A B}} & \text { for C. C. }\end{cases}
$$

and

$$
C_{3} \triangleq \begin{cases}\frac{\left(2^{R}-1\right)^{4}}{24\left(P_{A} P_{B} \lambda_{A D} \lambda_{B D}\right)^{2}} & \text { for D. C. } \\ \frac{2^{R}(R \ln 2)^{3}-3\left(2^{R}(R \ln 2)^{2}\right)+6\left(2^{R} R \ln 2-2^{R}+1\right)}{6\left(P_{A} P_{B} \lambda_{A D} \lambda_{B D}\right)^{2}} & \text { for C. C. }\end{cases}
$$

Using a similar technique as in Section V-A, taking the derivative of the objective function with respect to $\gamma$ and equating it to zero, we have:

$$
\begin{aligned}
\frac{\partial f_{2}(\gamma)}{\partial \gamma}= & \frac{4 C_{1}}{(1-\gamma)^{5}}+C_{2}\left[\frac{3}{\gamma(1-\gamma)^{4}}-\frac{1}{\gamma^{2}(1-\gamma)^{3}}\right]+ \\
& C_{3}\left[\frac{2}{(1-\gamma)^{3} \gamma^{2}}-\frac{2}{(1-\gamma)^{2} \gamma^{3}}\right]=0 \\
& \Longrightarrow \frac{4 C_{1}}{(1-\gamma)^{5}}+\frac{(4 \gamma-1) C_{2}}{\gamma^{2}(1-\gamma)^{4}}+\frac{(4 \gamma-2) C_{3}}{\gamma^{3}(1-\gamma)^{3}}=0
\end{aligned}
$$

which gives the following cubic equation:

$$
\begin{aligned}
& \left(4 C_{1}-4 C_{2}+4 C_{3}\right) \gamma^{3}+\left(5 C_{2}-10 C_{3}\right) \gamma^{2}+ \\
& \left(8 C_{3}-C_{2}\right) \gamma-2 C_{3}=0
\end{aligned}
$$

The optimal superposition ratio $\gamma_{o p t}^{1}$ can be obtained by solving for the roots of (16). Since the constant term $\left(-2 C_{3}\right)$ is negative, there is at least one positive real root for the cubic equation. Furthermore, we can show that the Hessian of (16) is always negative as $C_{1}, C_{2}$ and $C_{3}$ are bounded in the interval $(0,1]$ for high SNR values. Hence there is a unique positive real root for (16), which is the solution for $\gamma_{o p t}^{1}$. When $L=1$, $\gamma_{o p t}^{1}$ is independent of the spectral efficiency for the diversity combining case.

\section{Simulation Results}

In this section, first we compare the analytical packet error probability expressions derived in Section IV with empirical results. Then we present results illustrating the variation of the optimal superposition ratio as a function of the spectral efficiency for fixed values of the average channel gains and the average transmit power values. Finally, we compare the performance of the proposed retransmission scheme based on superposition modulation with the non-cooperative retransmission scheme and the retransmission scheme based on DF relaying discussed in Sections III-A and III-B. For all the simulation results, we have $\lambda_{A D}=\lambda_{B D}=\lambda_{A B}=\lambda$, $P_{A}=P_{B}=0 \mathrm{~dB}$. We define the average SNR (per channel use) as $\frac{\lambda}{\sigma^{2}}$. Modulation symbols have unit energy and the noise power $\sigma^{2}$ is set to 1 . The SNR is varied by varying $\lambda$ in the simulations.

\section{A. Analytical vs. Empirical Results}

Fig. 6 shows a comparison of analytical and empirical (using Monte Carlo simulation) results for $\mathrm{PEP}^{L}$ for both the diversity combining and the code combining cases. We have used $R=2 \mathrm{bpcu}, \lambda_{A D}=\lambda_{B D}=\lambda_{A B}$, and $P_{A}=P_{B}=0 \mathrm{~dB}$ in the simulation. Optimum superposition ratio values are used

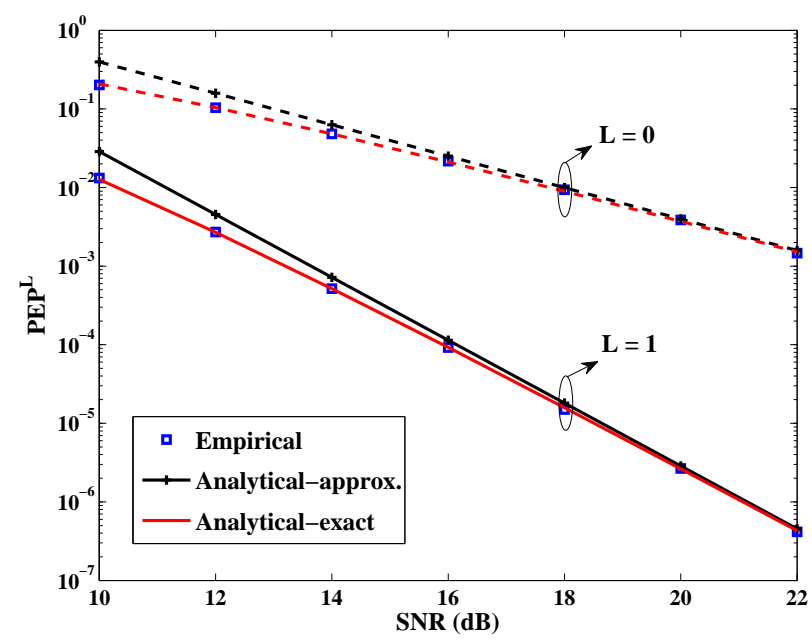

(a) With diversity combining.

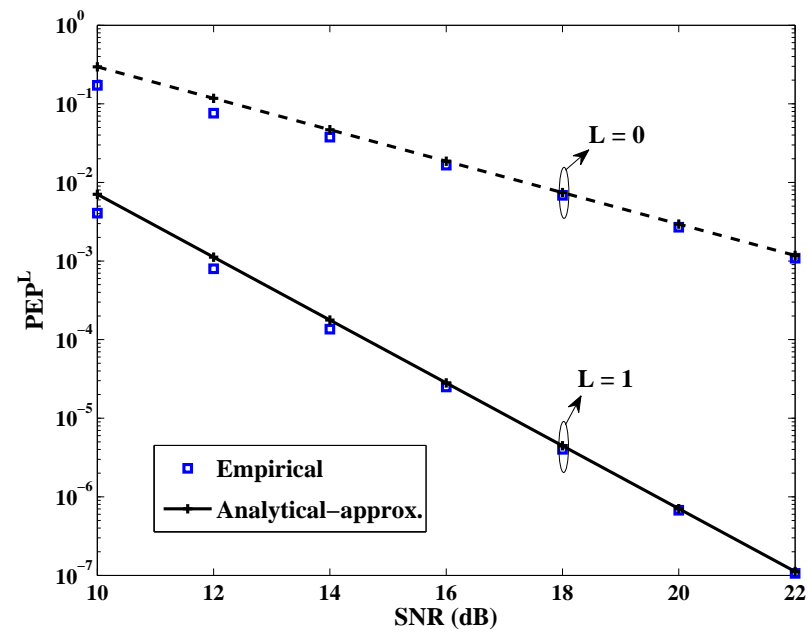

(b) With code combining.

Figure 6. Analytical and empirical performance comparison of the packet error probability for the proposed superposition modulation based relaying with retransmissions. Parameters for the simulation are $R=2 \mathrm{bpcu}, \lambda_{A D}=$ $\lambda_{B D}=\lambda_{A B}, P_{A}=P_{B}=0 \mathrm{~dB}$ and optimum $\gamma$ values obtained from Sections V-A and V-B are used for generating the plots.

for generating the curves. For the diversity combining case, the empirical (blue) curves are obtained by generating the channel gains randomly and numerically computing the probabilities in (4) and (7) (using Monte Carlo evaluation). For the "analyticalexact" (red) curves, we have used the expressions in (5) and (8) to compute the packet error probability by substituting the values of $P_{A}, P_{B}, \lambda_{A D}, \lambda_{B D}, \lambda_{A B}$ and $R$. The "analyticalapprox" (black) curves are high-SNR approximations obtained by neglecting the $O\left(\frac{1}{P^{3}}\right)$ and $O\left(\frac{1}{P^{5}}\right)$ terms in (6) and (9) respectively. For the code combining case, the empirical curves are obtained by Monte Carlo evaluation of the probabilities using (10) and (12), and the analytical approximations are the high-SNR asymptotes in (11) and (13). From Fig. 6(a), we see that analytical-exact result matches closely with the empirical result, proving the correctness of the expressions derived in this work. From Figures 6(a) and 6(b), we also 


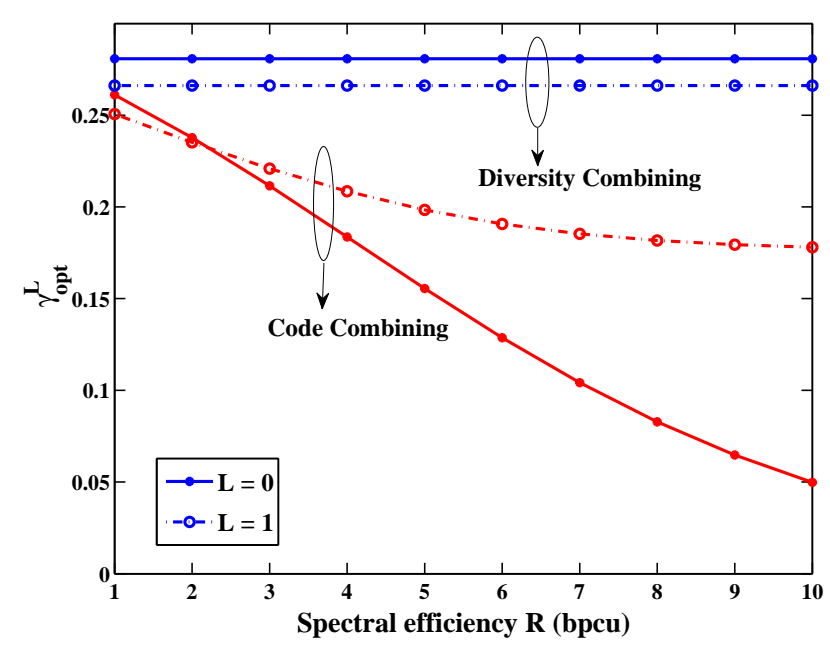

Figure 7. Optimal superposition ratio $\gamma$ as a function of spectral efficiency. The average channel gains for all the paths was set to 1 and the average transmit power for both the users was set to $30 \mathrm{~dB}$.

see that the analytical approximation is tight for SNR values higher than $15 \mathrm{~dB}$ for both the diversity combining and the code combining cases. We can also see the difference in the diversity order for the curves with $L=0$ and $L=1$.

\section{B. Optimal Superposition Ratio}

The variation of optimal $\gamma$ as function of $R$ for both the diversity combining and the code combining cases is shown in Fig. 7. From the figure, we see that for fixed average power and average channel gain values, the optimal $\gamma$ is independent of the spectral efficiency for the diversity combining case as discussed in Section V. For the code combining case, the optimum $\gamma$ is a function of $R$, and also a function of $L$ for higher values of $R$. Note that the optimum $\gamma$ values shown in the plot are slightly higher than the values obtained through simulations in [4]. The reason for this is that in our analysis here, we assumed a Gaussian code book and interference-free decoding at the destination. However in the simulations in [4], a fixed BPSK constellation was used and the effect of interference from the superimposed signal was also present during the decoding operation.

\section{Performance Comparison for Different $L$}

Fig. 8 shows an empirical comparison of $\mathrm{PEP}^{L}$ for different values of $L$ for both the diversity combining and the code combining cases. For the diversity combining case in Fig. 8(a), we considered a rate- $\frac{1}{2}$ convolutional code with a constraint length of 7 and generator function $G(133,171)^{3}$. The number of information bits $N_{I}$ is chosen to be 970 , and after appending CRC and convolutional encoding, we have 2000 coded bits for transmission. The number of channel uses for transmission $T$ is fixed to 2000. We transmit these coded bits using the three transmission methods as follows:

\footnotetext{
${ }^{3}$ Optimal in terms of free distance [25].
}

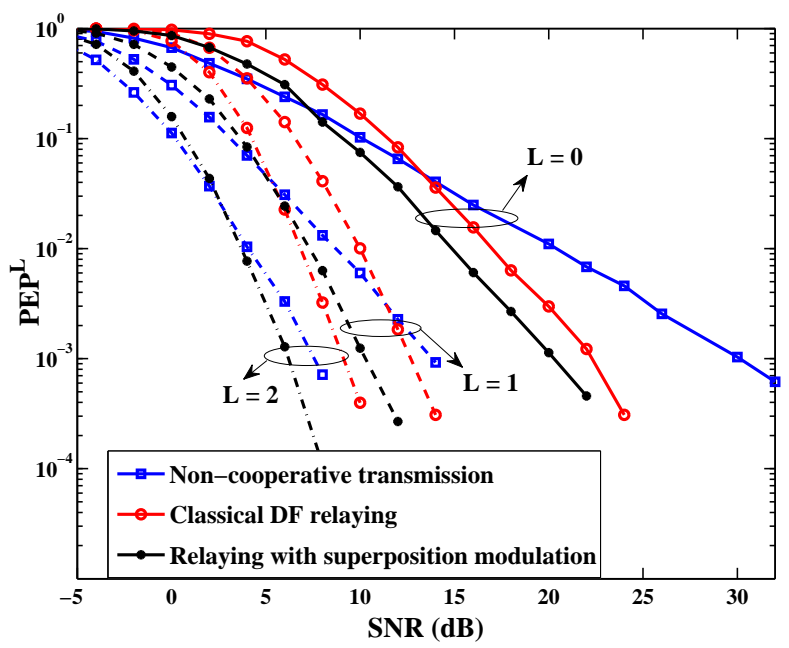

(a) With diversity combining. A rate- $\frac{1}{2}$ convolutional code with a constraint length 7 and the generator function of $G(133,171)$ is used for the simulation. The number of information bits $N_{I}$ is set to 970 . BPSK modulation is used for non-cooperative transmission and relaying with superposition modulation, and 4-PAM modulation is used for DF relaying case. The number of channel uses per transmission is $T=2000$.

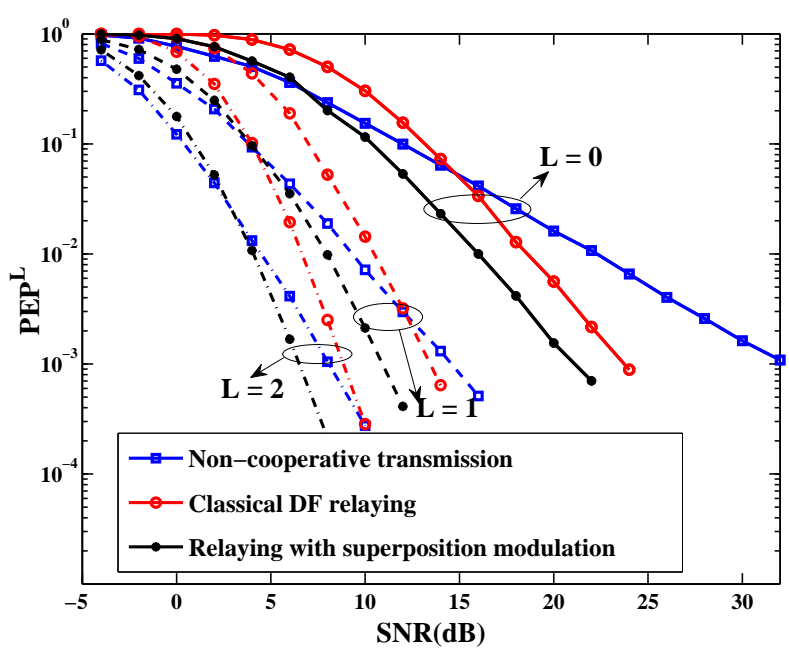

(b) With code combining. A rate- $\frac{1}{6}$ convolutional code with $N_{I}=972$ bits is used for the simulation. The constraint length for the code is 5 and the generator function is $G(37,35,27,33,25,35)$. BPSK modulation is used for non-cooperative transmission and relaying with superposition modulation, and 4-PAM modulation is used for DF relaying case. The number of channel uses per transmission is $T=2000$.

Figure 8. Link-level simulation results of packet error probability comparison for different $L$ for both the diversity combining and code combining cases.

- For non-cooperative transmission, 2000 coded bits are transmitted from node $\mathrm{A}$ in $T$ channel uses with BPSK modulation.

- For cooperation using DF relaying, 2000 coded bits are transmitted from node A in $\frac{T}{2}$ channel uses with 4-PAM modulation. If node B decodes node A's data successfully, it relays node A's bits in the remaining $\frac{T}{2}$ channel uses with 4-PAM modulation.

- For cooperation using superposition modulation based relaying, node A transmits its 2000 coded bits as well as 

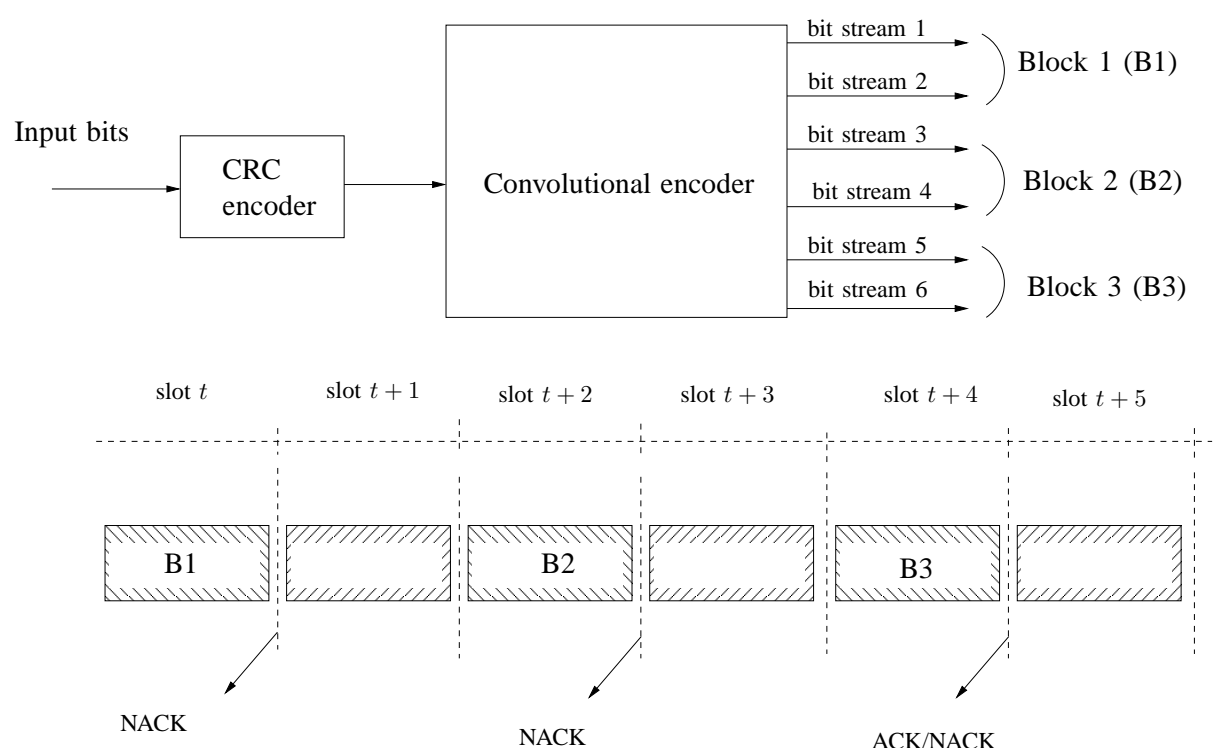

(a) Non-cooperative transmission.
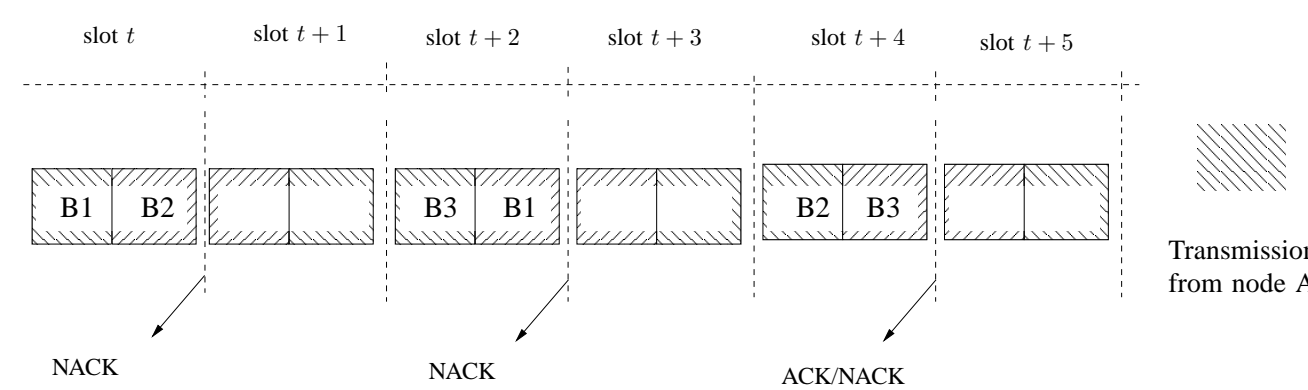

Transmission from node A

(b) Conventional DF relaying.

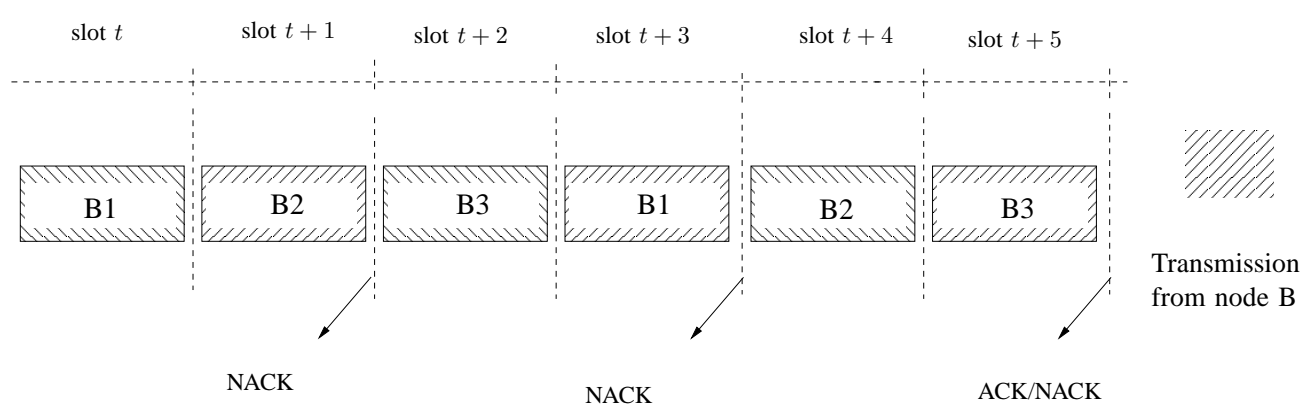

(c) Relaying with superposition modulation.

Figure 9. Simulation model for the code combining based retransmission scheme using the three transmission methods. Here for simplicity, we show only the transmission of data corresponding to node A. In the figure, as an example case, we assumed that the destination is not able to decode node A's data after one retransmission. 


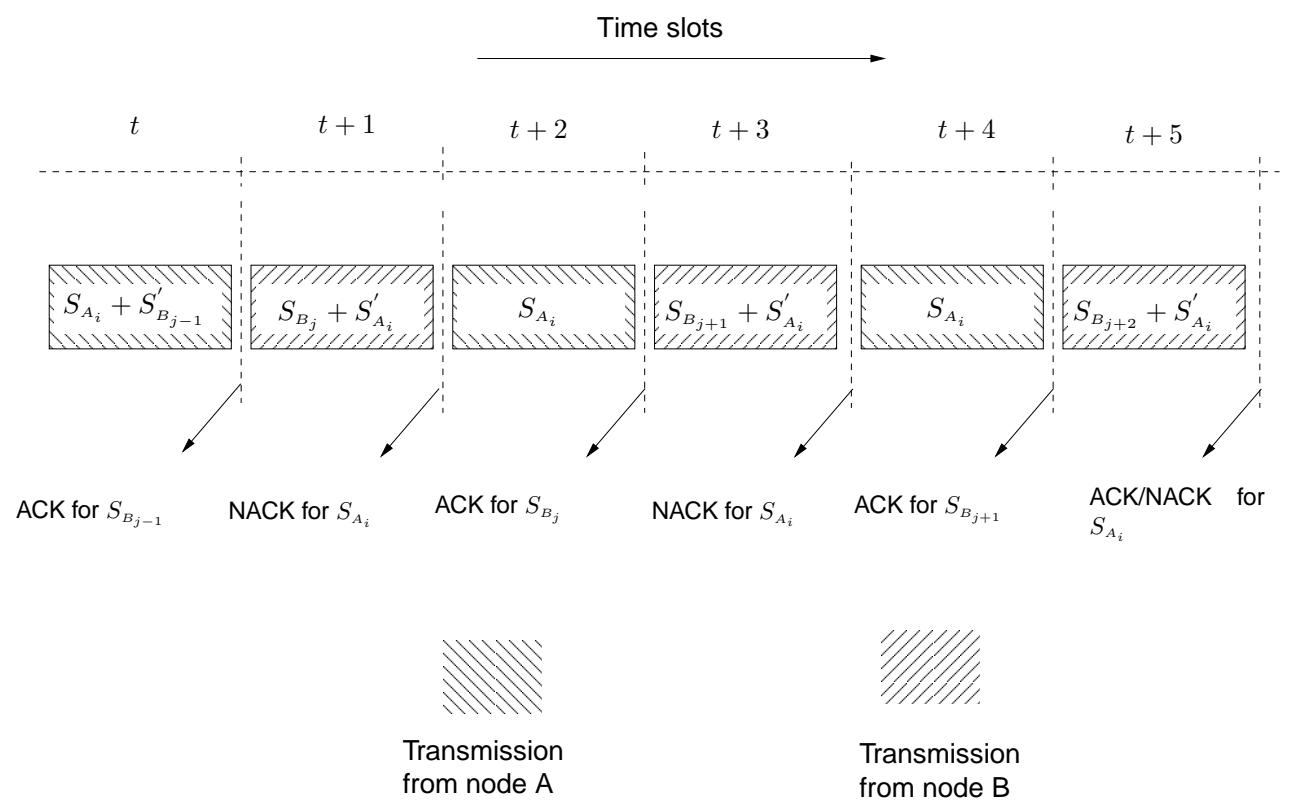

Figure 10. An example scenario showing the case in which node A does not use superposition during retransmission of its erroneous data packets.

node B's data in $T$ channel uses with BPSK modulation (before superposition operation) for its data. In the next time slot, if node B has successfully decoded node A's data, it relays node A's 2000 coded bits using BPSK modulation (before superposition operation) in $T$ channel uses.

- For the retransmissions also, the same modulation methods are used with repetition of coded bits.

We set the superposition ratio value to $\gamma=0.15$ and used the same value of $\gamma$ for all retransmissions. ${ }^{4}$ For $L=0$ and at a packet error probability of $10^{-3}$, relaying with superposition modulation has a performance gain of $2 \mathrm{~dB}$ compared to the classical DF relaying. This result is in agreement with the results in [4]. When $L=1$ and 2, the performance difference between relaying with superposition modulation and DF relaying is $2.4 \mathrm{~dB}$ and $2.9 \mathrm{~dB}$ respectively. Note that with $L=2$ and at a packet error probability of $10^{-3}$, the noncooperative retransmission scheme is performing better than the retransmission with DF relaying. This is due to the fact that the target spectral efficiency is double for the DF based relaying scheme and that its diversity advantage is coming into picture only for packet error probability values smaller than $10^{-4}$.

The result for the code combining case is shown in Fig. 8(b). For this simulation, we used a rate- $\frac{1}{6}$ convolutional code with a constraint length 5 and generator function $G(37,35,27,33,25,35)^{5}$. We transmitted the coded bits in an incremental redundancy fashion as shown in Fig. 9 for the three transmission methods. In Fig. 9, for simplicity, we showed only the transmission of data corresponding to node $A$. During the time slots $t+1, t+3$ and $t+5$ in the figure, for the retransmission schemes using non-cooperative

\footnotetext{
${ }^{4}$ Optimized through simulations. Note that the $\gamma_{o p t}$ shown in Fig. 7 is optimal only under the assumption described in Section IV and a Gaussian code book with an infinite block size.

${ }^{5}$ Optimal in terms of free distance [25].
}

transmission, and DF relaying, we showed the time slots as blank indicating that node A's data is not getting transmitted during these time slots. For the retransmission scheme using superposition modulation, during each time slot, we showed only the data corresponding to node A. With 972 information bits, after appending CRC and convolutional encoding, we have 6000 coded bits at the output (6 output streams and hence rate $-\frac{1}{6}$ ). These 6000 coded bits are divided into 3 blocks $\left(B_{1}, B_{2}\right.$ and $\left.B_{3}\right)$ of 2000 bits each. The number of channel uses per transmission $T$ is set to 2000. Transmission is done using these blocks in an incremental redundancy fashion as follows: ${ }^{6}$.

- As shown in Fig. 9(a), for the non-cooperative transmission, node A transmits the blocks $B_{1}, B_{2}$ and $B_{3}$ during each of its allocated time slots. As $T$ is set to 2000 , we use BPSK for modulating the coded bits.

- Fig. 9(b) shows transmission using the incremental redundancy based retransmission scheme with DF relaying. The $T$ channel uses are divided into $\frac{T}{2}$ channel uses each between the nodes A and B as they cooperate with each other. For this case, 4-PAM modulation is used as each block of 2000 bits should be transmitted using $\frac{T}{2}=1000$ channel uses. If $h_{A B}=0$, at the end of each transmission, the destination node will have the same amount of information as in the non-cooperative transmission scheme.

- Fig. 9(c) shows only the transmission of node A's data using the proposed retransmission scheme with incremental redundancy. In each transmission, 2000 coded bits are transmitted in $T$ channel uses. Here, node A's data

\footnotetext{
${ }^{6}$ Note that here we have used repetition of blocks for retransmission schemes with DF relaying and relaying using superposition modulation. This is not strictly optimal, however, combination of standard channel codes and repetition generally performs well for very low rates. In case of retransmission schemes with DF relaying and relaying using superposition modulation, ideally a rate-1/12 code should be used in this setup.
} 


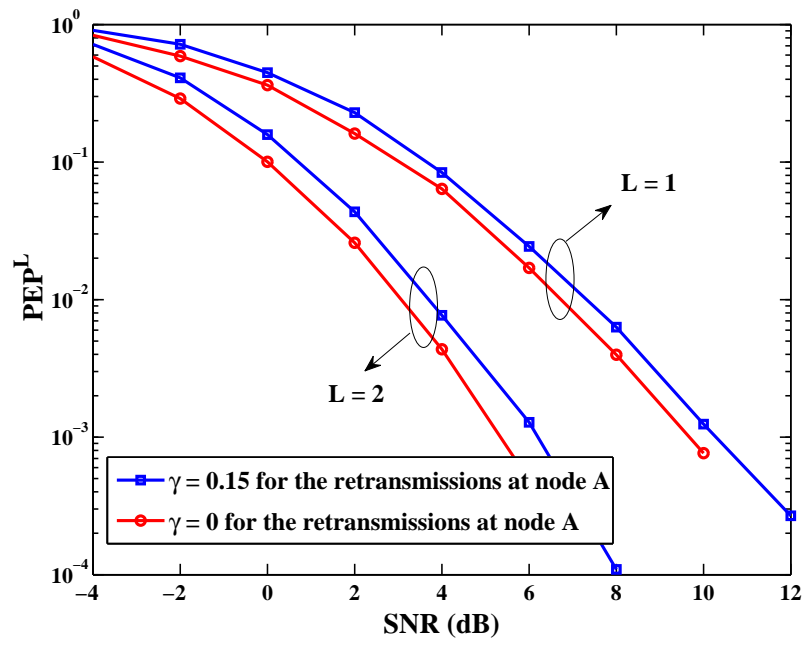

(a) With diversity combining.

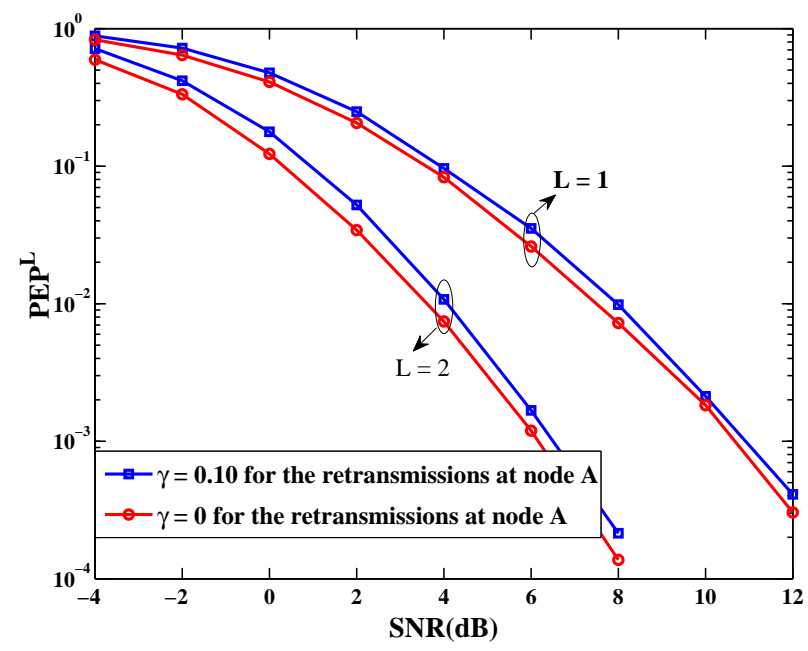

(b) With code combining.

Figure 11. Packet error probability comparison for the case when no superposition modulation is used at node A during retransmission of its erroneous packets. The simulation parameters are similar to the ones in Fig. 8.

gets transmitted both from node $\mathrm{A}$ as well as node $\mathrm{B}$ using superposition modulation. In this case also, when $h_{A B}=0$, this scheme reduces to the one with noncooperative transmission scheme (this is the basic idea of superposition modulation based cooperation scheme proposed in [4]).

For the simulation, the superposition ratio $\gamma$ is set to $0.1^{7}$. For $L=0$ and at a packet error probability of $10^{-3}$, relaying with superposition modulation shows a performance gain of $2.4 \mathrm{~dB}$ compared to the classical DF relaying. For $L=1$ and 2 , the gain for the relaying with superposition modulation over the DF relaying is $2.5 \mathrm{~dB}$.

\section{Varying Superposition Ratio for Retransmissions}

Next we present simulation results for the case in which node A transmits its own packet without superimposing the partners data during the retransmission. Node B still transmits node A's packet, which was in error, using superposition modulation. In a way this scenario reflects the case in which node A is not "fair" to node B. Fig. 10 shows an example of this scenario. As shown in figure, node A does not superpose node B's data during retransmission of its packet $S_{A_{i}}$ in time slots $t+2$ and $t+4$. However node B still superposes $S_{A_{i}}^{\prime}$ during time slots $t+3$ and $t+5$. The motivation for this simulation is that if the receiver could send additional feedback about the reliability of the previous transmissions, the transmitter could vary its superposition ratio (reduce the power allocated for partner's data) during the retransmissions. Fig. 11 shows a performance comparison of the packet error probability with diversity combining and code combining, for the case when node A does not superimpose node B's data during the retransmissions. We see that, when $L=2$, setting $\gamma=0$ for retransmissions at node A can only provide a marginal gain of about $0.5 \mathrm{~dB}$ at a packet error probability of $10^{-3}$ for both the diversity combining and the code combining cases. This result shows that even if the node, whose packets are in error is not "fair" to the cooperating node, the gains it can get are not significant.

\section{CONCLUSIONS}

The proposed retransmission scheme based on superposition modulation based relaying has superior performance compared to the retransmission schemes based on classical DF relaying and non-cooperative transmission. When only two retransmissions are allowed, at PEP values of interest, a non-cooperative retransmission scheme has better performance than the baseline retransmission scheme based on DF relaying. With the proposed retransmission scheme for the symmetric relaying scenario, nodes can still cooperate with each other even when their packets are received in error at the destination.

For the retransmission scheme with diversity combining, the optimal superposition ratio is independent of the spectral efficiency. For the retransmission scheme with code combining, with fixed average powers and average channel gains, the optimal superposition ratio depends on the maximum number of retransmissions allowed at high spectral efficiency values.

\section{APPENDIX A}

PROOF OF (5)

$\mathrm{PEP}_{\text {div }}^{0}$ can be derived as follows. From Equation (4), first we note that

$$
\begin{aligned}
\operatorname{Pr}\left(I_{A B}^{0}<R\right) & \triangleq \operatorname{Pr}\left(\log _{2}\left(1+P_{A}^{\prime} \alpha_{A B}^{0}\right)<R\right) \\
& =\operatorname{Pr}\left(\alpha_{A B}^{0}<\frac{2^{R}-1}{P_{A}^{\prime}}\right) \\
& =\int_{t=0}^{\frac{2^{R}-1}{P_{A}^{\prime}}} \frac{1}{\lambda_{A B}} \exp \left(-\frac{t}{\lambda_{A B}}\right) d t \\
& =1-\exp \left(X_{A B}\right)
\end{aligned}
$$

\footnotetext{
${ }^{7}$ Optimized through simulations.
} 
Similarly, we have

$$
\operatorname{Pr}\left(I_{A D}^{0}<R\right)=1-\exp \left(X_{A D}\right)
$$

and

$$
\operatorname{Pr}\left(I_{A B}^{0}>R\right)=\exp \left(X_{A B}\right)
$$

where $X_{A B}, X_{A D}$ are defined in Section IV-A1. Now $P_{M R C, 0} \triangleq \operatorname{Pr}\left(I_{M R C}\left(A D^{0}, B D^{0}\right)<R\right)$ can be simplified as

$$
\begin{aligned}
P_{M R C, 0}= & \operatorname{Pr}\left\{\left(P_{A}^{\prime} \alpha_{A D}^{0}+P_{B}^{\prime} \alpha_{B D}^{0}\right)<2^{R}-1\right\} \\
= & \int_{0}^{\frac{2^{R}-1}{P_{B}^{\prime}}} \operatorname{Pr}\left\{\alpha_{A D}^{0}<\frac{2^{R}-1-P_{B}^{\prime} t}{P_{A}^{\prime}}\right\} f_{\alpha_{B D}^{0}}(t) d t \\
= & \int_{0}^{\frac{2^{R}-1}{P_{B}^{\prime}}}\left[1-\exp \left(\frac{P_{B}^{\prime} t+1-2^{R}}{P_{A}^{\prime} \lambda_{A D}}\right)\right] \times \\
& \frac{1}{\lambda_{B D}} \exp \left(-\frac{t}{\lambda_{B D}}\right) d t \\
= & 1-\exp \left(\frac{1-2^{R}}{P_{B}^{\prime} \lambda_{B D}}\right)-\exp \left(\frac{1-2^{R}}{P_{A}^{\prime} \lambda_{A D}}\right) \times \\
& \underbrace{\frac{1}{\lambda_{B D}} \int_{0}^{\frac{2^{R}}{P_{B}^{\prime}}} \exp \left(-t\left[\frac{1}{\lambda_{B D}}-\frac{P_{B}^{\prime}}{P_{A}^{\prime} \lambda_{A D}}\right]\right) d t}_{\triangleq Z}
\end{aligned}
$$

The term $Z$ can be further simplified to arrive at

$$
Z= \begin{cases}\frac{1}{\delta}\left[1-\exp \left(X_{B D} \delta\right)\right] & \text { if } \delta \neq 0 \\ -X_{B D} & \text { if } \delta=0\end{cases}
$$

Using (17)-(21) in (4), we arrive at (5).

APPENDiX B

PROOF OF (8)

From (7),

$$
\begin{aligned}
P_{M R C, 1} & \triangleq \operatorname{Pr}\left(I_{M R C}\left(A B^{0}, A B^{1}\right)<R\right) \\
& =\operatorname{Pr}\left\{\left(\alpha_{A B}^{0}+\alpha_{A B}^{1}\right)<\frac{2^{R}-1}{P_{A}^{\prime}}\right\} \\
& \stackrel{(a)}{=} \operatorname{Pr}\left\{\alpha_{A B}^{*}<\frac{2^{R}-1}{P_{A}^{\prime}}\right\} \\
& =\int_{0}^{\frac{2^{R}-1}{P_{A}^{\prime}}} \frac{t}{\lambda_{A B}^{2}} \exp \left(-\frac{t}{\lambda_{A B}}\right) d t \\
& =1-\exp \left(X_{A B}\right)\left(1-X_{A B}\right)
\end{aligned}
$$

In (a), $\alpha_{A B}^{*}=\alpha_{A B}^{0}+\alpha_{A B}^{1}$, which is the sum of two independent exponential random variables with the same mean $\lambda_{A B}$. Hence its distribution is given by $f_{\alpha_{A B}^{*}}(t)=\frac{t}{\lambda_{A B}^{2}} \exp \left(-\frac{t}{\lambda_{A B}}\right), t \geq$ 0 . Similarly, we have

$$
\operatorname{Pr}\left(I_{M R C}\left(A D^{0}, A D^{1}\right)<R\right)=1-\exp \left(X_{A D}\right)\left(1-X_{A D}\right)
$$

Defining $P_{1} \triangleq \operatorname{Pr}\left(I_{A B}^{0}<R, I_{M R C}\left(A B^{0}, A B^{1}\right)>R\right)$, we have

$$
\begin{aligned}
P_{1} & =\operatorname{Pr}\left\{\left(\alpha_{A B}^{0}+\alpha_{A B}^{1}\right)>\frac{2^{R}-1}{P_{A}^{\prime}}, \alpha_{A B}^{0}<\frac{2^{R}-1}{P_{A}^{\prime}}\right\} \\
& =\int_{0}^{\frac{2^{R}-1}{P_{A}^{\prime}}} \operatorname{Pr}\left\{\alpha_{A B}^{1}>\frac{2^{R}-1}{P_{A}^{\prime}}-t\right\} \frac{1}{\lambda_{A B}} \exp \left(-\frac{t}{\lambda_{A B}}\right) d t \\
& =\int_{0}^{\frac{2^{R}-1}{P_{A}^{\prime}}} \exp \left(\frac{1-2^{R}}{P_{A}^{\prime} \lambda_{A B}}+\frac{t}{\lambda_{A B}}\right) \frac{1}{\lambda_{A B}} \exp \left(-\frac{t}{\lambda_{A B}}\right) d t \\
& =-X_{A B} \exp \left(X_{A B}\right)
\end{aligned}
$$

Defining $P_{M R C, 2} \triangleq \operatorname{Pr}\left(I_{M R C}\left(A D^{0}, A D^{1}, B D^{1}\right)<R\right)$, we have

$$
\begin{aligned}
P_{M R C, 2}= & \operatorname{Pr}\left\{\left(\alpha_{A D}^{*} P_{A}^{\prime}+\alpha_{B D}^{1} P_{B}^{\prime}\right)<2^{R}-1\right\} \\
= & \int_{0}^{\frac{2^{R}-1}{P_{B}^{\prime}}} \operatorname{Pr}\left\{\alpha_{A D}^{*}<\frac{2^{R}-1-t P_{B}^{\prime}}{P_{A}^{\prime}}\right\} \times \\
& \frac{1}{\lambda_{B D}} \exp \left(-\frac{t}{\lambda_{B D}}\right) d t \\
= & \int_{0}^{\frac{2^{R}-1}{P_{B}^{\prime}}}\left[1-\exp \left(\frac{t P_{B}^{\prime}+1-2^{R}}{P_{A}^{\prime} \lambda_{A D}}\right) \times\right. \\
& \left.\left(1+\frac{t P_{B}^{\prime}+1-2^{R}}{P_{A}^{\prime} \lambda_{A D}}\right)\right] \frac{1}{\lambda_{B D}} \exp \left(-\frac{t}{\lambda_{B D}}\right) d t
\end{aligned}
$$

$P_{M R C, 2}$ in (25) can be further simplified as in (26) shown at the top of the next page. The integrals in (26) can be further simplified to arrive at:

$$
\begin{aligned}
& Z_{1}=1-\exp \left(X_{B D}\right) \\
& Z_{2}= \begin{cases}\frac{1}{\delta}\left[1-\exp \left(X_{B D} \delta\right)\right] & \text { if } \delta \neq 0 \\
-X_{B D} & \text { if } \delta=0\end{cases} \\
& Z_{3}= \begin{cases}\lambda_{B D}\left[\exp \left(X_{B D} \delta\right)\left(\frac{X_{B D}}{\delta}-\frac{1}{\delta^{2}}\right)+\frac{1}{\delta^{2}}\right] & \text { if } \delta \neq 0 \\
-\frac{X_{B D}\left(2^{R}-1\right)}{2 P_{B}^{\prime}} & \text { if } \delta=0\end{cases}
\end{aligned}
$$

$\operatorname{Pr}\left(I_{A B}^{0}>R\right)$ is as given in (19). Defining $P_{M R C, 3} \triangleq$ $\operatorname{Pr}\left(I_{M R C}\left(A D^{0}, A D^{1}, B D^{0}, B D^{1}\right)<R\right)$, we have

$$
\begin{aligned}
P_{M R C, 3}= & \operatorname{Pr}\left\{\left[\alpha_{A D}^{*} P_{A}^{\prime}+\alpha_{B D}^{*} P_{B}^{\prime}\right]<2^{R}-1\right\} \\
= & \int_{0}^{\frac{2^{R}-1}{P_{B}^{\prime}}} \operatorname{Pr}\left\{\alpha_{A D}^{*}<\frac{2^{R}-1-t P_{B}^{\prime}}{P_{A}^{\prime}}\right\} \times \\
& \frac{t}{\lambda_{B D}^{2}} \exp \left(-\frac{t}{\lambda_{B D}}\right) d t \\
= & \int_{0}^{\frac{2^{R}-1}{P_{B}^{\prime}}}\left[1-\exp \left(\frac{t P_{B}^{\prime}+1-2^{R}}{P_{A}^{\prime} \lambda_{A D}}\right) \times\right. \\
& \left.\left(1+\frac{t P_{B}^{\prime}+1-2^{R}}{P_{A}^{\prime} \lambda_{A D}}\right)\right] \frac{t}{\lambda_{B D}^{2}} \exp \left(-\frac{t}{\lambda_{B D}}\right) d t
\end{aligned}
$$

$P_{M R C, 3}$ in (28) can be further simplified as in (29) shown on top of the next page. 


$$
\begin{aligned}
P_{M R C, 2}= & \underbrace{\int_{0}^{\frac{2^{R}-1}{P_{B}^{\prime}}} \frac{1}{\lambda_{B D}} \exp \left(-\frac{t}{\lambda_{B D}}\right) d t}_{\triangleq Z_{1}}+\left[X_{A D} \exp \left(X_{A D}\right)-\exp \left(X_{A D}\right)\right] \underbrace{\int_{0}^{\frac{2^{R}-1}{P_{B}^{\prime}}} \frac{1}{\lambda_{B D}} \exp \left(-t\left(\frac{1}{\lambda_{B D}}-\frac{P_{B}^{\prime}}{P_{A}^{\prime} \lambda_{A D}}\right)\right) d t}_{\triangleq Z_{2}} \\
& +\frac{P_{B}^{\prime} \exp \left(X_{A D}\right)}{P_{A}^{\prime} \lambda_{A D}} \underbrace{\int_{0}^{\frac{2^{R}-1}{P_{B}^{\prime}}} \frac{t}{\lambda_{B D}} \exp \left(-t\left(\frac{1}{\lambda_{B D}}-\frac{P_{B}^{\prime}}{P_{A}^{\prime} \lambda_{A D}}\right)\right) d t}_{\triangleq Z_{3}}
\end{aligned}
$$

$$
\begin{aligned}
P_{M R C, 3} & =\underbrace{\int_{0}^{\frac{2^{R}-1}{P_{B}^{\prime}}} \frac{t}{\lambda_{B D}^{2}} \exp \left(-\frac{t}{\lambda_{B D}}\right) d t}_{\triangleq Y_{1}}+\left[X_{A D} \exp \left(X_{A D}\right)-\exp \left(X_{A D}\right)\right] \underbrace{\int_{0}^{\frac{2^{R}-1}{P_{B}^{\prime}}} \frac{t}{\lambda_{B D}^{2}} \exp \left(-t\left(\frac{1}{\lambda_{B D}}-\frac{P_{B}^{\prime}}{P_{A}^{\prime} \lambda_{A D}}\right)\right) d t}_{\triangleq Y_{2}} \\
& +\frac{P_{B}^{\prime} \exp \left(X_{A D}\right)}{P_{A}^{\prime} \lambda_{A D}} \underbrace{\int_{0}^{\frac{2^{R}-1}{P_{B}^{\prime}}} \frac{t^{2}}{\lambda_{B D}^{2}} \exp \left(-t\left(\frac{1}{\lambda_{B D}}-\frac{P_{B}^{\prime}}{P_{A}^{\prime} \lambda_{A D}}\right)\right) d t}_{\triangleq Y_{3}}
\end{aligned}
$$

$$
\begin{aligned}
& Y_{1}=1-\exp \left(X_{B D}\right)\left(1-X_{B D}\right) \\
& Y_{2}= \begin{cases}\left(\exp \left(X_{B D} \delta\right)\left(\frac{X_{B D}}{\delta}-\frac{1}{\delta^{2}}\right)+\frac{1}{\delta^{2}}\right) & \text { if } \delta \neq 0 \\
\frac{X_{B D}^{2}}{2} & \text { if } \delta=0\end{cases} \\
& Y_{3}= \begin{cases}\lambda_{B D}\left[\exp \left(X_{B D} \delta\right)\left(-\frac{X_{B D}^{2}}{\delta}+\frac{2 X_{B D}}{\delta^{2}}-\frac{2}{\delta^{3}}\right)+\frac{2}{\delta^{3}}\right] & \text { if } \delta \neq 0 \\
\frac{X_{B D}^{2}\left(2^{R}-1\right)}{3 P_{B}^{\prime}} & \text { if } \delta=0\end{cases}
\end{aligned}
$$

The integrals in (29) can be simplified as in (30) shown on top of this page. Using (22)-(30) in (7), we obtain the expression for $\mathrm{PEP}_{\text {div }}^{1}$ as in (8).

\section{APPENDIX C \\ PROOF OF (11)}

From (10), the term $P_{c c, 0} \triangleq \operatorname{Pr}\left(I_{A D}^{0}+I_{B D}^{0}<R\right)=$ $\operatorname{Pr}\{(x+y)<R\}$, where $x=\log _{2}\left(1+\alpha_{A D}^{0} P_{A}^{\prime}\right)$ and $y=$ $\log _{2}\left(1+\alpha_{B D}^{0} P_{B}^{\prime}\right)$ can be simplified as follows. Using the following distributions for $x$ and $y$

$$
\begin{aligned}
& f_{x}(t)=\frac{\ln 2}{P_{A}^{\prime} \lambda_{A D}} \exp \left(\frac{1-2^{t}}{P_{A}^{\prime} \lambda_{A D}}\right) 2^{t}, \quad t \geq 0 \text { and } \\
& f_{y}(t)=\frac{\ln 2}{P_{B}^{\prime} \lambda_{B D}} \exp \left(\frac{1-2^{t}}{P_{B}^{\prime} \lambda_{B D}}\right) 2^{t}, \quad t \geq 0
\end{aligned}
$$

we can write

$$
\begin{aligned}
P_{c c, 0} & =\int_{0}^{R} \operatorname{Pr}\{x<R-t\} f_{y}(t) d t \\
& =\int_{0}^{R}\left(1-\exp \left(\frac{1-2^{R-t}}{P_{A}^{\prime} \lambda_{A D}}\right)\right) f_{y}(t) d t \\
& =\int_{0}^{R} f_{y}(t) d t-\int_{0}^{R} \exp \left(\frac{1-2^{R-t}}{P_{A}^{\prime} \lambda_{A D}}\right) f_{y}(t) d t \\
& =\int_{0}^{R} f_{y}(t) d t-\frac{\ln 2}{P_{B}^{\prime} \lambda_{B D}} \exp \left(\frac{1}{P_{A}^{\prime} \lambda_{A D}}+\frac{1}{P_{B}^{\prime} \lambda_{B D}}\right) \times
\end{aligned}
$$

$$
\begin{aligned}
& \int_{0}^{R} \exp \left(-\frac{2^{R-t}}{P_{A}^{\prime} \lambda_{A D}}-\frac{2^{t}}{P_{B}^{\prime} \lambda_{B D}}\right) 2^{t} d t \\
& \stackrel{(\mathrm{b})}{=} \underbrace{\int_{0}^{R} f_{y}(t) d t}_{\triangleq L_{1}}-\frac{\ln 2}{P_{B}^{\prime} \lambda_{B D}} \exp \left(\frac{1}{P_{A}^{\prime} \lambda_{A D}}+\frac{1}{P_{B}^{\prime} \lambda_{B D}}\right) \times \\
& \underbrace{\int_{0}^{R}\left(1-\frac{2^{R-t}}{P_{A}^{\prime} \lambda_{A D}}-\frac{2^{t}}{P_{B}^{\prime} \lambda_{B D}}\right) 2^{t} d t}_{\triangleq L_{2}}
\end{aligned}
$$

In (b), we have used the expansion $\exp (x)=1+x+O\left(x^{2}\right)$. The integrals in (31) can be simplified to get

$$
\begin{aligned}
& L_{1}=1-\exp \left(\frac{1-2^{R-t}}{P_{A}^{\prime} \lambda_{A D}}\right) \\
& L_{2}=\frac{2^{R}-1}{\ln 2}-\frac{2^{2 R}-1}{2 \ln 2 P_{B}^{\prime} \lambda_{B D}}-\frac{R 2^{R}}{P_{A}^{\prime} \lambda_{A D}}
\end{aligned}
$$

Using (32) in (31) and simplifying using a series expansion, we get

$$
P_{c c, 0}=\frac{2^{R} R \ln 2-2^{R}+1}{P_{A}^{\prime} P_{B}^{\prime} \lambda_{A D} \lambda_{B D}}+O\left(\frac{1}{P^{3}}\right)
$$

Now using (17)-(19) with a series expansion and (33), from (10) we arrive at (11). 


$$
\begin{aligned}
P_{c c, 2}= & \int_{0}^{R} \int_{0}^{R-t} \int_{0}^{R-t-u} \operatorname{Pr}\{w<R-t-u-v\} f_{x}(t) f_{y}(u) f_{z}(v) d v d u d t \\
= & \int_{0}^{R} \int_{0}^{R-t} \int_{0}^{R-t-u}\left(1-\exp \left(\frac{1-2^{R-t-u-v}}{P_{B}^{\prime} \lambda_{B D}}\right)\right) f_{x}(t) f_{y}(u) f_{z}(v) d v d u d t \\
= & \int_{0}^{R} \int_{0}^{R-t} \int_{0}^{R-t-u} f_{x}(t) f_{y}(u) f_{z}(v) d v d u d t- \\
= & \underbrace{\int_{0}^{R} \int_{0}^{R-t} \int_{0}^{R-t} f_{x}(t) f_{y}(u) d u d t}_{\triangleq M_{1}}-\underbrace{\left.\frac{1-2^{R-t-u}}{P_{B}^{\prime} \lambda_{B D}}\right) f_{x}(t) f_{y}(u) d u d t-}_{\int_{0}^{\int_{B}} \int_{0}^{R-t} \exp \left(\frac{1-2^{R-t-u-v}}{P_{B}^{\prime} \lambda_{B D}}\right) f_{x}(t) f_{y}(u) f_{z}(v) d v d u d t} \\
& \underbrace{\int_{0}^{R-t} \int_{0}^{R}}_{\int_{0}^{R-t-u} \exp \left(\frac{1-2^{R-t-u-v}}{P_{B}^{\prime} \lambda_{B D}}\right) f_{x}(t) f_{y}(u) f_{z}(v) d v d u d t}
\end{aligned}
$$

\section{ApPENDIX D \\ PROOF OF (13)}

In (12), $\operatorname{Pr}\left(I_{A B}^{0}+I_{A B}^{1}<R\right)$ and $\operatorname{Pr}\left(I_{A D}^{0}+I_{A D}^{1}<R\right)$ can be simplified similarly as in Appendix $\mathrm{C}$, to get

$$
\begin{aligned}
& \operatorname{Pr}\left(I_{A B}^{0}+I_{A B}^{1}<R\right)=\frac{2^{R} R \ln 2-2^{R}+1}{\left(P_{A}^{\prime} \lambda_{A B}\right)^{2}}+O\left(\frac{1}{P^{3}}\right) \\
& \operatorname{Pr}\left(I_{A D}^{0}+I_{A D}^{1}<R\right)=\frac{2^{R} R \ln 2-2^{R}+1}{\left(P_{A}^{\prime} \lambda_{A D}\right)^{2}}+O\left(\frac{1}{P^{3}}\right)
\end{aligned}
$$

Considering the term

$$
\begin{aligned}
P_{c c, 1} & \triangleq \operatorname{Pr}\left(I_{A B}^{0}<R, I_{A B}^{0}+I_{A B}^{1}>R\right) \\
& =\operatorname{Pr}(x<R, x+y>R)
\end{aligned}
$$

with $x=I_{A B}^{0}$ and $y=I_{A B}^{1}$, we have

$$
\begin{aligned}
P_{c c, 1} & =\int_{0}^{R} \operatorname{Pr}\{y>R-t\} f_{x}(t) d t \\
& =\int_{0}^{R} \exp \left(\frac{1-2^{R-t}}{P_{A}^{\prime} \lambda_{A B}}\right) f_{x}(t) d t \\
& =\frac{\ln 2}{P_{A}^{\prime} \lambda_{A B}} \exp \left(\frac{2}{P_{A}^{\prime} \lambda_{A B}}\right) \int_{0}^{R} \exp \left(-\frac{2^{R-t}+2^{t}}{P_{A}^{\prime} \lambda_{A B}}\right) 2^{t} d t \\
& =\frac{\ln 2}{P_{A}^{\prime} \lambda_{A B}} \exp \left(\frac{2}{P_{A}^{\prime} \lambda_{A B}}\right) \int_{0}^{R}\left(1-\frac{2^{R-t}+2^{t}}{P_{A}^{\prime} \lambda_{A B}}\right) 2^{t} d t \\
& =-X_{A B}+O\left(\frac{1}{P^{2}}\right)
\end{aligned}
$$

Now considering the term

$$
\begin{aligned}
P_{c c, 2} & \triangleq \operatorname{Pr}\left(I_{A D}^{0}+I_{A D}^{1}+I_{B D}^{0}+I_{B D}^{1}<R\right) \\
& =\operatorname{Pr}(x+y+z+w<R)
\end{aligned}
$$

with $x=I_{A D}^{0}, y=I_{A D}^{1}, z=I_{B D}^{0}$ and $w=I_{B D}^{1}$, we can write $P_{c c, 2}$ as in (36) shown at the top of this page. Using a series expansion and simplifying, the expressions for $M_{1}, M_{2}$ and $M_{3}$ in (36) can simplified to arrive at the expressions in (37)-(39) shown on top of the next page. This yields the final expression for $P_{c c, 2}$ as shown in (40).
The term $P_{c c, 3} \triangleq \operatorname{Pr}\left(I_{A D}^{0}+I_{A D}^{1}+I_{B D}^{1}<R\right)$ is equal to $M_{1}-M_{2}$, and hence

$$
P_{c c, 3}=\frac{\left(2^{R}(R \ln 2)^{2}-2\left(2^{R} R \ln 2-2^{R}+1\right)\right)}{2\left(P_{A}^{\prime} \lambda_{A D}\right)^{2} P_{B}^{\prime} \lambda_{B D}}+O\left(\frac{1}{P^{4}}\right)
$$

From (34), (35), (40) and (41), we arrive at the PEP expression in (13).

\section{REFERENCES}

[1] J. N. Laneman, D. N. Tse, and G. W. Wornell, "Cooperative diversity in wireless networks: efficient protocol and outage," IEEE Trans. Inform. Theory, vol. 50, pp. 3062-3080, Dec. 2004.

[2] A. Sendonaris, E. Erkip, and B. Aazhang, "User cooperation diversity, part I: system description," IEEE Trans. Commun., vol. 51, pp. 19271938, Nov. 2003.

[3] T. E. Hunter and A. Hedayat, "Cooperative communication in wireless networks," IEEE Commun. Mag., pp. 74-80, Oct. 2004.

[4] E. G. Larsson and B. Vojcic, "Cooperative transmit diversity based on superposition modulation," IEEE Communications Letters, vol. 9, pp. 778-780, Sep. 2005.

[5] L. Xiao, T. E. Fuja, J. Kliewer and D. J. Costello, "A network coding approach to cooperative diversity," IEEE Trans. Inf. Theory, vol. 53, no. 10, pp. 3714-3722, Oct. 2007.

[6] T. Yang, J. Yuan, "Performance of iterative decoding for superposition modulation-based cooperative transmission," IEEE Trans. Wireless Commun., vol. 9, pp. 51-59, Jan. 2010.

[7] Z. Ding, T. Ratnarajah, and C. C. F. Cowan, " On the diversitymultiplexing tradeoff for wireless cooperative multiple access systems," IEEE Trans. Signal. Process., vol. 55, no. 9, pp. 4627-4638, Sep. 2007.

[8] C. Hasan and Ü. Aygölü, "An incremental relaying approach for superposition modulated cooperative transmission," Proc. of IEEE WCNC, Apr. 2009.

[9] I. Krikidis, "Analysis and optimization issues for superposition modulation in cooperative networks," IEEE Trans. Veh. Tech., vol. 58. no.9, pp. 4837-4847, Nov. 2009.

[10] K. Ishii, "Superposition modulated cooperative diversity for half-duplex scenario," Journal of Communications, vol. 2, no. 7, pp. 20-27, Dec. 2007.

[11] S. Lin, D. J. Costello, Jr., and M. J. Miller, "Automatic-repeat-request error control schemes," IEEE Commun. Mag., vol. 22, pp. 5-16, Dec. 1984.

[12] T. Beigbeder et al., "The Effects of loss and latency on user performance in unreal tournament 2003", Proc. of ACM workshop on network and system support for games, pp. 144-151, Portland, USA, Sept. 2004.

[13] G. Yu, Z. Zhang, and P. Qiu, "Cooperative ARQ in wireless networks: protocols description and performance analysis," Proc. of IEEE ICC, vol. 8, pp. 3608-3614, Jun. 2006. 


$$
\begin{aligned}
& M_{1}= \frac{2^{R} R \ln 2-2^{R}+1}{\left(P_{A}^{\prime} \lambda_{A D}\right)^{2}}+\frac{2\left(2^{R} R \ln 2\right)-2^{2 R}+1}{\left(P_{A}^{\prime} \lambda_{A D}\right)^{3}}+ \\
& \frac{6\left(2^{2 R} R \ln 2\right)+24\left(2^{R} R \ln 2\right)+18\left(2^{R}\right)-27\left(2^{2 R}\right)+2\left(2^{3 R}\right)+7}{12\left(P_{A}^{\prime} \lambda_{A D}\right)^{4}}+O\left(\frac{1}{P^{5}}\right) \\
& M_{2}= M_{1}+\frac{2\left(2^{R} R \ln 2-2^{R}+1\right)-2^{R}(R \ln 2)^{2}}{2\left(P_{A}^{\prime} \lambda_{A D}\right)^{2} P_{B}^{\prime} \lambda_{B D}}+\frac{2^{2 R}-2\left(2^{R}\right)+1-2^{R}(R \ln 2)^{2}}{2\left(P_{A}^{\prime} P_{B}^{\prime} \lambda_{A D} \lambda_{B D}\right)^{2}}+ \\
& \frac{2^{2 R}-2\left(2^{R}\right)+1-2^{R}(R \ln 2)^{2}}{\left(P_{A}^{\prime} \lambda_{A D}\right)^{3} P_{B}^{\prime} \lambda_{B D}}+\left(\frac{1}{P^{5}}\right) \\
& M_{3}=-\frac{2\left(2^{R} R \ln 2-2^{R}+1\right)-2^{R}(R \ln 2)^{2}}{2\left(P_{A}^{\prime} \lambda_{A D}\right)^{2} P_{B}^{\prime} \lambda_{B D}} \frac{2^{2 R}-2\left(2^{R}\right)+1-2^{R}(R \ln 2)^{2}}{\left(P_{A}^{\prime} \lambda_{A D}\right)^{3} P_{B}^{\prime} \lambda_{B D}}+ \\
& 6\left(2^{R}(R \ln 2)^{2}-2^{R}(R \ln 2)\right)-2^{R}(R \ln 2)^{3}+12\left(2^{R}\right)-3\left(2^{2 R}\right)-9 \\
& 6\left(P_{A}^{\prime} P_{B}^{\prime} \lambda_{A D} \lambda_{B D}\right)^{2}
\end{aligned}
$$

$$
P_{c c, 2}=\frac{2^{R}(R \ln 2)^{3}-3\left(2^{R}(R \ln 2)^{2}\right)+6\left(2^{R} R \ln 2-2^{R}+1\right)}{6\left(P_{A}^{\prime} P_{B}^{\prime} \lambda_{A D} \lambda_{B D}\right)^{2}}+O\left(\frac{1}{P^{5}}\right)
$$

[14] A. Agustin, J. Vidal and O. Muñoz, "Analytical resource optimization for the DF relay-assisted transmission under HARQ," Proc. of IEEE VTC, Apr. 2009.

[15] B. Zhao and M. C. Valenti, "Practical relay networks: a generalization of hybrid-ARQ," IEEE J. Select. Areas. Commun., vol. 23, pp. 7-18, Jan. 2005.

[16] I. Krikidis, "Distributed truncated ARQ protocol for cooperative diversity networks," IET Communications, vol. 1, pp. 1212-1217, Dec. 2007.

[17] K. Zheng, L. Hu, W. Wang and L. Huang, "Performance analysis of HARQ transmission in cooperative DF relaying systems," Wireless Personal Communications, vol. 55, pp. 441-455, Nov. 2010.

[18] M. Costa, "Writing on dirty paper," IEEE Trans. Inform. Theory, vol. 29, pp. 439-441, May 1983.

[19] X. Qiu and K. Chawla "On the performance of adaptive modulation in cellular systems," IEEE Trans. Commun., vol. 47, pp. 884-895, Jun. 1999.

[20] R. Zhang and L. Hanzo, "Superposition-coding-aided multiplexed hybrid ARQ scheme for improved end-to-end transmission efficiency," IEEE Trans. Vech. Tech., vol 58. no.8, pp. 4681-4686, Oct. 2009.

[21] F. Takahashi and K. Higuchi, "HARQ for predetermined-rate multicast channel," Proc. of IEEE VTC, May 2010.

[22] T. V. K. Chaitanya and E. G. Larsson, "Retransmission strategies for symmetric relaying using superposition modulation," Proc. of IEEE VTC, Sep. 2010.

[23] M. N. Khormuji and E. G. Larsson, "Cooperative transmission based on decode-and-forward relaying with partial repetition coding," IEEE Trans. on Wireless Commun., vol. 8, pp. 1716-1725, Apr. 2009.

[24] J. Hagenauer, "Rate-compatible punctured convolutional codes (RCPC codes) and their applications," IEEE Trans. Commun., vol. 36, pp. 389400, Apr. 1988.

[25] J. G. Proakis and M. Salehi, Digital Communication (5th Ed.), McGrawHill, 2008.

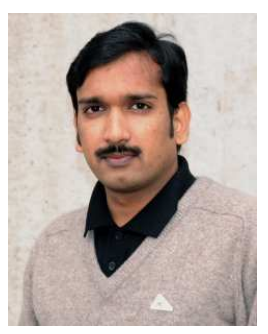

Tumula V. K. Chaitanya received his Masters degree in Telecommunications Engineering from Indian Institute of Science, Bangalore, in June 2005. From July 2005 to December 2008, he was with Beceem Communications Pvt. Ltd., Bangalore, as a Senior Design Engineer. Currently, he is a Ph.D student at the Division of Communication Systems, Department of Electrical Engineering, Linköping University (LiU), Sweden. His research interests include Signal Processing Algorithms for Wireless Communications, Resource Allocation in Wireless Networks and Cooperative Communications.

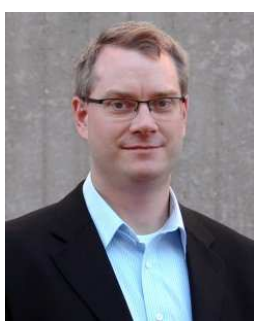

Erik G. Larsson received his $\mathrm{Ph} . \mathrm{D}$. degree from Uppsala University, Sweden, in 2002. Since 2007, he is Professor and Head of the Division for Communication Systems in the Department of Electrical Engineering (ISY) at Linköping University (LiU) in Linköping, Sweden. He has previously been Associate Professor (Docent) at the Royal Institute of Technology (KTH) in Stockholm, Sweden, and Assistant Professor at the University of Florida and the George Washington University, USA.

His main professional interests are within the areas of wireless communications and signal processing. He has published some 70 journal papers on these topics, he is co-author of the textbook SpaceTime Block Coding for Wireless Communications (Cambridge Univ. Press, 2003) and he holds 10 patents on wireless technology.

$\mathrm{He}$ is Associate Editor for the IEEE Transactions on Communications and he has previously been Associate Editor for several other IEEE journals. $\mathrm{He}$ is a member of the IEEE Signal Processing Society SAM and SPCOM technical committees. He is active in conference organization, most recently as the Technical Chair of the Asilomar Conference on Signals, Systems and Computers 2012 and Technical Program co-chair of the International Symposium on Turbo Codes and Iterative Information Processing 2012. 